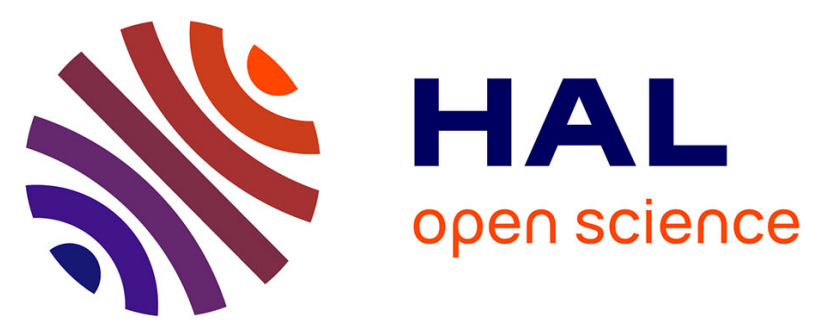

\title{
Vectorisation of agrochemicals via amino acid carriers: influence of the spacer arm structure on the phloem mobility of phenylpyrrole conjugates in the Ricinus system
}

Sophie Marhadour, Hanxiang Wu, Wen Yang, Cécile Marivingt-Mounir, Jean-Louis Bonnemain, Jean-François Chollet

\section{To cite this version:}

Sophie Marhadour, Hanxiang Wu, Wen Yang, Cécile Marivingt-Mounir, Jean-Louis Bonnemain, et al.. Vectorisation of agrochemicals via amino acid carriers: influence of the spacer arm structure on the phloem mobility of phenylpyrrole conjugates in the Ricinus system: Vectorization of agrochemicals via amino acid carriers in the Ricinus system. Pest Management Science, 2017, 73 (9), pp.1972-1982. $10.1002 /$ ps.4575. hal-02115808

\section{HAL Id: hal-02115808 https://hal.science/hal-02115808}

Submitted on 30 Apr 2019

HAL is a multi-disciplinary open access archive for the deposit and dissemination of scientific research documents, whether they are published or not. The documents may come from teaching and research institutions in France or abroad, or from public or private research centers.
L'archive ouverte pluridisciplinaire HAL, est destinée au dépôt et à la diffusion de documents scientifiques de niveau recherche, publiés ou non, émanant des établissements d'enseignement et de recherche français ou étrangers, des laboratoires publics ou privés. 


\section{Vectorization of agrochemicals via amino acid carriers: influence of the spacer arm structure on the phloem mobility of phenylpyrrole conjugates in the Ricinus system}

Vectorization of agrochemicals via amino acid carriers in the Ricinus system

Sophie Marhadour ${ }^{\mathrm{a}, \mathrm{w}}$, Hanxiang $\mathrm{Wu}^{\mathrm{a}, \mathrm{c}, \text { }}$, Wen Yang ${ }^{\mathrm{b}}$, Cécile Marivingt-Mounir ${ }^{\mathrm{a}}$, Jean-Louis Bonnemain $^{\mathrm{c}}$ and Jean-François Chollet ${ }^{\mathrm{a}, *}$

${ }^{a}$ Institut de Chimie des Milieux et des Matériaux de Poitiers (IC2MP), Unité Mixte de Recherche CNRS 7285, Université de Poitiers, 4 rue Michel Brunet, TSA 51106, F-86073

Poitiers cedex 9, France

${ }^{b}$ Guizhou Tea Reasearch Institute, Guizhou Academy of Agricultural Science, Guiyang, Guizhou 550009, China.

${ }^{c}$ Laboratoire Écologie et Biologie des Interactions, Unité Mixte de Recherche CNRS 7267, Université de Poitiers, 3 rue Jacques Fort, TSA 51106, F-86073 Poitiers Cedex 9, France

* Corresponding author: Tel. fax: +33 549453965

E-mail address: jean.franc.chollet@univ-poitiers.fr

¥ These authors contributed equally to the paper

E-mail addresses : sophie.marhadour@gmail.com; hanxiang.wu@univ-poitiers.fr; yangwen3409@126.com; cecile.marivingt.mounir@univ-poitiers.fr; jl.bonnemain@yahoo.fr; jean.francois.chollet@univ-poitiers.fr 


\section{ABSTRACT}

BACKGROUND: Excessive agrochemical use causes significant threats to environmental safety and human health. Reducing pesticide use without reducing yield is necessary for sustainable agriculture. Therefore we developed a vectorization strategy to enhance agrochemical delivery through plant amino acid carriers.

RESULTS: In addition to a fenpiclonil conjugate recently described, three new amino acid conjugates were synthesized by coupling fenpiclonil to an L- $\alpha$-amino acid. Phloem mobility of these conjugates which exhibit different structures of the spacer arm introduced between fenpiclonil and the $\alpha$-amino acid function, was studied using the Ricinus model. Conjugate $\mathbf{L}$ 14 which contains a triazole ring with the shortest amino acid chain showed the best phloem systemicity among the four conjugates. By contrast, removing the triazole ring in the spacer arm did not improve systemicity. L-14 exhibited phloem systemicity at all reported pH values (pHs from 5.0 to 6.5) of the foliar apoplast, while acidic derivatives of fenpiclonil were translocated only at $\mathrm{pH}$ values near 5.0.

CONCLUSION: The conjugates were recognized by a pH-dependent transporter system and translocated at distance in the phloem. They exhibited a broader ability to phloem systemicity than fenpiclonil acidic derivatives within the $\mathrm{pH}$ value range of the foliar apoplast.

\section{KEYWORDS}

Phenylpyrroles, Fungicide, Conjugate systemicity, Phloem transport, Sustainable agriculture, Amino acid carrier 


\section{INTRODUCTION}

To protect crops from damage by weeds, pathogens and animal pests, agrochemicals remain an important part of current agricultural systems to secure food production in addition to improvement of cultural practices, biological control of pathogens and pests, and development of new strategies confering genetic resistance. ${ }^{1-3}$ But, meanwhile, the discovery of optimal efficacy and low-use-rate pesticides to reduce the impact on human health and environment have become urgent and necessary. Prodrug approaches in pharmaceutical research have been developed to enhance bioavailability through targeted drug delivery and accumulation, ${ }^{4}$ which have set a good example for agrochemical design. ${ }^{5}$ Ensuring pesticide effective delivery to sites of action presents a promising strategy which not only can keep the dose at the lowest possible level, but also can improve the biological activity of active ingredient. For example, profungicides, which are likely to concentrate in plant phloem tissues, can be applied to control vascular diseases ${ }^{6}$ where the most existent fungicides are unable to access. One of attractive prodrug approach is to conjugate a drug with endogenous substrates, so that the resulting molecules can be recognized and transported by peptide, amino acid or glucose transporters. ${ }^{7-15}$

Carrier-mediated processes have been proved to be a promising approach to enhance the uptake and vectorisation of pesticides by utilizing plant sugar or amino acid transporters as well. ${ }^{16,17}$ Both glucose and amino acid pesticide conjugates were reported to involve carriermediated uptake and displayed phloem mobility. ${ }^{18-20}$ This propesticide strategy could be applied to develop phloem mobile insecticides, which are needed to control root or vascular pests. ${ }^{17,19}$ Similarly, profungicides designed to move at long distance in the phloem tissue could be applied to control vascular or root diseases of herbaceous cultures, i.e. tissues or organ where the current commercialized fungicides are unable to access after foliar application. $^{21}$

Fenpiclonil, a non-phloem mobile fungicide was selected in a recent past as a model compound because of the numerous possibilities of adding substituents, notably carboxylic groups ${ }^{22,23}$ at various sites in the molecule. In a recent study on phloem-mobile profungicides, a fenpiclonil-glucose and a fenpiclonil-glutamic acid conjugates were synthesized via click chemistry to compare the ability of amino acid and sugar carrier systems for profungicide uptake and phloem mobility. ${ }^{24}$ The results of systemicity test indicated that the $L$-amino acid promoiety was dramatically more favourable to phloem mobility than the $D$-amino acid and $D$-glucose promoieties. However, the structure-activity relationship between conjugates and 
amino acid carrier systems has not yet been extended to other parameters such as the structure of the $L$-amino acid promoiety and the possible effect of the triazole spacer. The optimal characterization of chemical structures for recognition and translocation of amino acid conjugate remains unknown.

In the present work, we describe the synthesis and phloem systemicity of three new amino acid conjugates in which fenpiclonil is attached to $L$ - $\alpha$-amino acids exhibiting different chain lengths and with or without a triazole spacer. In order to avoid any interaction of the antifungal moiety with the active site of the carrier, we added a spacer linker between the amino acid function and the antifungal compound. The structure notably included a 1,2,3triazole ring connected to the antifungal moiety via an amide bond. The 1,2,3-triazole, as a peptide bond isostere, is often described to improve stability, absorption or aqueous solubility of different bioconjugation compounds. ${ }^{25,26}$ In drug discovery, the replacement of amide bonds in the backbone of peptides by 1,4-disubstituted 1,2,3-triazole lead to peptidomimetics with retained receptor affinity and improved tumor-targeting capabilities. ${ }^{27}$ Using click chemistry, this linker presents the advantage to be synthesized easily and quickly with good yields. ${ }^{28}$ Phloem systemicity studies were conducted using Ricinus seedling cotyledons which function as the small intestine wall. ${ }^{29,30}$ The aim of the second part of this paper is: 1- to investigate the influence of different chain length and type of the spacer arm (i.e. the linkage between fenpiclonil and the $\alpha$-aminoacid function) on phloem mobility and 2 - to compare the phloem systemicity of the most mobile amino acid conjugate with the acidic derivatives of fenpiclonil at various external $\mathrm{pH}$ values with a special attention for apoplast $\mathrm{pH}$ values measured in mature leaf tissues of various plant species.

\section{MATERIAL AND METHODS}

\subsection{Synthesis}

Some reactions were carried out under nitrogen. All reactions were monitored by TLC analysis using Merck silica gel 60F-254 thin-layer plates. Column chromatography was carried out on silica gel Merck $60(0.015-0.04 \mathrm{~mm})$. Melting points were determined on an Electrothermal IA 9200 melting point apparatus and are uncorrected. ${ }^{1} \mathrm{H}$ and ${ }^{13} \mathrm{C}$ NMR spectra were performed in DMSO-d6 using a Bruker AVANCE $400 \mathrm{MHz}$ spectrometer. DEPT 135 and ${ }^{1} \mathrm{H}-{ }^{13} \mathrm{C}$ experiments were used to confirm the NMR peak assignements. Chemical shifts are reported as $\delta$ values in parts per million (ppm) relative to tetramethylsilane as internal standard and coupling constants $(J)$ are given in hertz $(\mathrm{Hz})$. The following abbreviations are 
used to describe peak patterns when appropriate: s (singlet), d (doublet), t (triplet), $\mathrm{q}$ (quartet), m (multiplet). High-resolution mass spectra were obtained on a Bruker Qtof Maxis Impact spectrometer.

\subsection{Plant material}

Castor bean seeds (Ricinus communis L. cv Sanguineus), obtained from Graines Girerd et Fils (Le Thor, France) were placed in wet cotton wool for $24 \mathrm{~h}$ at $27^{\circ} \mathrm{C} \pm 1^{\circ} \mathrm{C}$ prior to sowing in vermiculite watered with tap water. Seedlings were grown in a humid atmosphere $(80 \% \pm$ $5 \%$ ) at $27^{\circ} \mathrm{C} \pm 1^{\circ} \mathrm{C}$. As the brush-border of the small intestine, cotyledon tissues exhibit a high ability to take up small nutrient molecules, but from an external compartment, namely the endosperm (Fig. 1A). ${ }^{30,31}$

\subsection{Phloem sap collection and analysis}

The sap collection method was similar to that already described (Figs. 1B, C, D, E). ${ }^{32}$ The phloem sap was analyzed by HPLC after dilution with UHQ grade water $(1+9 \mathrm{v} / \mathrm{v})$. We employed reversed phase chromatography using an Ascentis Express RP-amide C16 column (length $250 \mathrm{~mm}$, internal diameter $4.6 \mathrm{~mm}, 5 \mu \mathrm{M}$ ) (Supelco, Bellefonte, PA) in accordance with the procedure set out in Table 1. Results were processed with PC 1000 software v3.5 from Thermo Fisher Scientific (Courtabœuf, France).

\subsection{Chemicals}

The compounds to be added to incubation solutions were from Acros Organics (Noisy-leGrand, France) (4-morpholinoethanesulfonic acid [MES], 2-[4-(2-hydroxyethyl)-1piperazine]ethanesulfonic acid [HEPES]).

\subsection{Physicochemical properties}

Physicochemical properties and descriptors were predicted using ACD / Labs Percepta 2015 release (Build 2726) software from Advanced Chemistry Development, Inc. (Toronto, Canada). The calculated properties (Table 2) were chosen according to their influence on passive membrane transport in plants. ${ }^{33}$ 


\section{RESULTS AND DISCUSSION}

\subsection{Synthesis of amino acid conjugates}

In a previous work, we described the three-step synthesis of compound L-13 which associated fenpiclonil, a fungicide from the phenylpyrrole family, to L-glutamic acid via a spacer group including a 1,2,3-triazole ring. This conjugate was obtained by coupling the azido derivative 3 of protected glutamic acid with the propargyl derivative of fenpiclonil 10. Then the deprotection of the $\alpha$-amino acid function of the resulting coumpound $\mathbf{1 1} \mathbf{R}_{\mathbf{1}}$ gave the desired conjugate L-13 (Fig. 2). ${ }^{24}$ A similar synthetic route was adapted in this work to obtain the conjugates L-14 and L-15. The procedure involves a CuAAC ("Copper(I)-catalyzed AzideAlkyne Cycloaddition") permitting the introduction of a 1,2,3-triazole ring by click chemistry. ${ }^{34-36}$ In order to study the influence of the triazole ring on phloem mobility, we synthesized compound L-16 which does not have this heterocycle in its structure. In this case, the strategy was to form an amide bond using a coupling reagent. ${ }^{37-40}$ The different processes are outlined in Fig. 2.

\subsubsection{Obtaining azido derivatives from protected amino acid (Fig. 2; compounds 7, 8)}

We first started with the synthesis of azido derivatives $\mathrm{N}_{3}-\mathrm{R}_{\mathrm{X}}$ from commercially available protected amino acid. The conversion of primary amines to azides via diazo-transfer occured using trifluoromethanesulfonyl azide $\left(\mathrm{TfN}_{3}\right)$, an efficient organic-soluble reactive described by Cavender and Shiner. ${ }^{41} \mathrm{TfN}_{3}$ is not available commercially and was prepared immediately prior to use. It was made by reacting trifluoromethanesulfonic anhydride with sodium azide in a mixture water-dichloromethane. ${ }^{42-44}$ Thus, the trifluoromethanesulfonyl azide $\mathbf{4}$ allowed to convert protected $\alpha$-aminoacid compounds 5 or $\mathbf{6}$ respectively into the desired azides $\mathbf{7}$ and $\mathbf{8}$ in a one-pot reaction ${ }^{45}$ in presence of potassium carbonate and copper (II) sulfate pentahydrate in a methanol-water medium.

Experimental procedure for the synthesis of 4-azido-2-[(tert-butoxycarbonyl)amino]butanoic acid (7) and 6-azido-2-[(tert-butoxycarbonyl)amino]hexanoic acid (8)

To a solution of sodium azide $(6.52 \mathrm{~g}, 100.0 \mathrm{mmol}, 10$ equiv) in a mixture dichloromethane/water (42 mL, 2/1) trifluoromethanesulfonic anhydride (3.33 mL, 20.0 mmol, 2 equiv) was added at $0^{\circ} \mathrm{C}$. The reaction mixture was allowed to warm to room temperature and was stirred for $2 \mathrm{~h}$. The water layer was extracted with dichloromethane and the combined organic layers were washed with saturated sodium carbonate solution. The resulting solution of $\mathrm{TfN}_{3} 4$ in dichloromethane was added slowly to a solution of protected 
amino acid $N$ - $\alpha$-Boc- $L$-2,4-diaminobutyric acid 5 (2.18 g, $10.0 \mathrm{mmol}, 1$ equiv) or $N$ - $\alpha$-Boc- $L$ lysine 6 (2.47 g, $10.0 \mathrm{mmol}, 1$ equiv), potassium carbonate ( $2.08 \mathrm{~g}, 15.0 \mathrm{mmol}, 1.5$ equiv) and copper (II) sulfate pentahydrate $(25.0 \mathrm{mg}, 0.10 \mathrm{mmol}, 0.01$ equiv) in a mixture methanol/water $(96 \mathrm{~mL}, 2 / 1)$. The reaction mixture was stirred at room temperature for $12 \mathrm{~h}$ and the organic solvents were evaporated under reduced pressure. The aqueous layer was used directly for the next step.

\subsubsection{Coupling azido derivatives $N_{3}-R_{x}$ and the propargyl derivative of fenpiclonil 10 by click chemistry (Fig. 2; compounds $11 \boldsymbol{R}_{\mathbf{2}}, \mathbf{1 1} \boldsymbol{R}_{\mathbf{3}}$ )}

For this step, we prepared a key intermediate of fenpiclonil substituted with an alkyne function (compound 10), as previously described. ${ }^{24}$ Thus, we considered a 1,3-cycloaddition by click chemistry with an appropriate coupling partner, the azido derivatives $\mathbf{7}$ and $\mathbf{8}$ giving the 1,2,3-triazole ring as a spacer group to obtain targeted compounds $\mathbf{1 1} \mathbf{R}_{\mathbf{2}}$ and $\mathbf{1 1} \mathbf{R}_{\mathbf{3}}$. ${ }^{34,46}$ This reaction was carried out using as catalyst the active $\mathrm{Cu}(\mathrm{I})$, generated from $\mathrm{Cu}(\mathrm{II})$ salts with sodium ascorbate as the reducing agent in a mixture tert-butanol-water. In these conditions the copper-catalyzed reaction allowed the synthesis of the 1,4-disubstituted regioisomers specifically. ${ }^{47}$ The compounds $\mathbf{1 1} \mathbf{R}_{\mathbf{2}}$ and $\mathbf{1 1} \mathbf{R}_{\mathbf{3}}$ were obtained in moderate yields, $40 \%$ and $44 \%$ respectively. In a general approach, this pathway offers the possibility of a wide diversity for further structure-activity relationship study.

Experimental procedure for the synthesis of derivatives $11 \boldsymbol{R}_{\mathbf{2}}$ and $\mathbf{1 1} \boldsymbol{R}_{\mathbf{3}}$ (Fig. 3)

To a solution of compound $10(3.47 \mathrm{~g}, 10.0 \mathrm{mmol}, 1$ equiv) in tert-butanol $(28 \mathrm{~mL})$, the solution of compound $\mathbf{7}$ or compound $\mathbf{8}$ previously prepared was added. Then, a solution of copper (II) sulfate pentahydrate (500 mg, $2.0 \mathrm{mmol}, 0.2$ equiv) and $L$-ascorbic acid sodium salt (794 mg, $4.0 \mathrm{mmol}, 0.4$ equiv) in water $(28 \mathrm{~mL})$ was added to the reaction mixture. The resulting solution was heated at $50^{\circ} \mathrm{C}$ for $2.5 \mathrm{~h}$. After cooling to room temperature, the resulting mixture was diluted with ethyl acetate. The organic layer was extracted, washed with saturated ammonium chloride solution and brine, dried over $\mathrm{MgSO}_{4}$, filtered and concentrated under vacuum. The crude product was purified by silica gel column chromatography using ethyl acetate/methanol (9: 1) as eluent to obtain respectively compound $\mathbf{1 1} \mathbf{R}_{\mathbf{2}}$ as a pink powder (2.4 g, $40 \%$ yield) or compound $11 \mathbf{R}_{3}$ as a beige powder ( $2.7 \mathrm{~g}, 44 \%$ yield).

2-(Tert-butoxycarbonylamino)-4-(4-\{[2-(3-cyano-4-(2,3-dichlorophenyl)-1H-pyrrol-1yl)propanamido]methyl $\}-1 H-1,2,3$-triazol-1-yl)butanoic acid (11R $\mathbf{2})$

$\mathrm{Rf}=0.60$ (ethyl acetate/methanol: $5 / 5) ; \mathrm{Mp}=163-164{ }^{\circ} \mathrm{C} .{ }^{1} \mathrm{H}$ NMR $\left(400 \mathrm{MHz}, \mathrm{DMSO}-d_{6}\right): \delta$ $13.01(\mathrm{~s}, 1 \mathrm{H}, \mathrm{OH}), 8.83\left(\mathrm{t}, 1 \mathrm{H},{ }^{3} \mathrm{~J}=5.2 \mathrm{~Hz}, \mathrm{NH}\right), 7.98\left(\mathrm{~s}, 1 \mathrm{H}, \mathrm{H}_{\mathrm{d}}\right), 7.88\left(\mathrm{~d}, 1 \mathrm{H},{ }^{4} \mathrm{~J}=2.2 \mathrm{~Hz}\right.$, 
$\left.\mathrm{H}_{2}\right), 7.68\left(\mathrm{dd}, 1 \mathrm{H},{ }^{3} J=7.3 \mathrm{~Hz},{ }^{4} J=2.3 \mathrm{~Hz}, \mathrm{H}_{8}\right), 7.49-7.43\left(\mathrm{~m}, 2 \mathrm{H}, \mathrm{H}_{6}\right.$ and $\left.\mathrm{H}_{7}\right), 7.30\left(\mathrm{~d}, 1 \mathrm{H},{ }^{4} J\right.$ $\left.=2.2 \mathrm{~Hz}, \mathrm{H}_{5}\right), 7.19(\mathrm{~s}, 1 \mathrm{H}, \mathrm{NH}), 4.98\left(\mathrm{q}, 1 \mathrm{H},{ }^{3} J=7.0 \mathrm{~Hz}, \mathrm{H}_{\mathrm{b}}\right), 4.43-4.32\left(\mathrm{~m}, 4 \mathrm{H}, \mathrm{H}_{\mathrm{c}}\right.$ and $\left.\mathrm{H}_{\mathrm{e}}\right)$, 3.86-3.82 (m, 1H, $\left.\mathrm{H}_{\mathrm{g}}\right), 2.26-2.08\left(\mathrm{~m}, 2 \mathrm{H}, \mathrm{H}_{\mathrm{f}}\right), 1.65\left(\mathrm{~d}, 3 \mathrm{H},{ }^{3} \mathrm{~J}=7.0 \mathrm{~Hz}, \mathrm{H}_{\mathrm{a}}\right), 1.42\left(\mathrm{~s}, 9 \mathrm{H}, \mathrm{H}_{\mathrm{h}}\right)$. ${ }^{13} \mathrm{C}$ NMR (100 MHz, DMSO- $\left.d_{6}\right): \delta 170.33(\mathrm{C}=\mathrm{O}), 169.04(\mathrm{C}=\mathrm{O}), 156.55(\mathrm{C}=\mathrm{O}), 144.94(\mathrm{C})$, $133.98(\mathrm{C}-\mathrm{Cl}), 132.47(\mathrm{C}-\mathrm{Cl}), 130.26\left(\mathrm{C}_{7}\right.$ and $\left.\mathrm{C}\right), 129.71\left(\mathrm{C}_{8}\right), 129.03\left(\mathrm{C}_{2}\right), 128.21\left(\mathrm{C}_{6}\right)$, $123.01\left(\mathrm{C}_{\mathrm{d}}\right), 122.17\left(\mathrm{C}_{4}\right), 122.08\left(\mathrm{C}_{5}\right), 116.05(\mathrm{CN}), 91.98\left(\mathrm{C}_{3}\right), 78.18(\mathrm{C}), 59.75\left(\mathrm{C}_{\mathrm{g}}\right), 57.57$ $\left(\mathrm{C}_{\mathrm{b}}\right), 46.86\left(\mathrm{C}_{\mathrm{e}}\right), 34.47\left(\mathrm{C}_{\mathrm{c}}\right), 31.72\left(\mathrm{C}_{\mathrm{f}}\right), 28.18\left(3 \mathrm{C}_{\mathrm{h}}\right), 18.28\left(\mathrm{C}_{\mathrm{a}}\right)$. HRMS (ESI, $\left.\mathrm{CH}_{3} \mathrm{CN}\right): \mathrm{m} / \mathrm{z}$ calculated for $\mathrm{C}_{26} \mathrm{H}_{29} \mathrm{Cl}_{2} \mathrm{~N}_{7} \mathrm{O}_{5}[\mathrm{M}+\mathrm{Na}]^{+} 612.1505, m / z$ found 612.1501 .

2-(Tert-butoxycarbonylamino)-6-(4-\{[2-(3-cyano-4-(2,3-dichlorophenyl)-1H-pyrrol-1-

yl)propanamido]methyl $\}-1 H$-1,2,3-triazol-1-yl)hexanoic acid (11R $\mathbf{3})$

$\mathrm{Rf}=0.13$ (ethyl acetate/methanol: $9 / 1) ; \mathrm{Mp}=142-143{ }^{\circ} \mathrm{C} .{ }^{1} \mathrm{H}$ NMR $\left(400 \mathrm{MHz}\right.$, DMSO- $\left.d_{6}\right): \delta$ $12.56(\mathrm{~s}, 1 \mathrm{H}, \mathrm{OH}), 8.87\left(\mathrm{t}, 1 \mathrm{H},{ }^{3} J=5.3 \mathrm{~Hz}, \mathrm{NH}\right), 7.97\left(\mathrm{~s}, 1 \mathrm{H}, \mathrm{H}_{\mathrm{d}}\right), 7.88\left(\mathrm{~d}, 1 \mathrm{H},{ }^{4} J=2.2 \mathrm{~Hz}\right.$ $\left.\mathrm{H}_{2}\right), 7.68\left(\mathrm{dd}, 1 \mathrm{H},{ }^{3} J=7.1 \mathrm{~Hz},{ }^{4} J=2.5 \mathrm{~Hz}, \mathrm{H}_{8}\right), 7.48-7.44\left(\mathrm{~m}, 2 \mathrm{H}, \mathrm{H}_{6}\right.$ and $\left.\mathrm{H}_{7}\right), 7.30\left(\mathrm{~d}, 1 \mathrm{H},{ }^{4} J\right.$ $\left.=2.2 \mathrm{~Hz}, \mathrm{H}_{5}\right), 6.64\left(\mathrm{~d}, 1 \mathrm{H},{ }^{3} J=7.6 \mathrm{~Hz}, \mathrm{NH}\right), 4.99\left(\mathrm{q}, 1 \mathrm{H},{ }^{3} J=7.0 \mathrm{~Hz}, \mathrm{H}_{\mathrm{b}}\right), 4.38-4.32(\mathrm{~m}, 4 \mathrm{H}$, $\mathrm{H}_{\mathrm{c}}$ and $\left.\mathrm{H}_{\mathrm{e}}\right)$, 3.87-3.81 (m, 1H, $\left.\mathrm{H}_{\mathrm{i}}\right), 1.85-1.52\left(\mathrm{~m}, 7 \mathrm{H}, \mathrm{H}_{\mathrm{a}}, \mathrm{H}_{\mathrm{f}}\right.$ and $\left.\mathrm{H}_{\mathrm{h}}\right), 1.40-1.31\left(\mathrm{~m}, 11 \mathrm{H}, \mathrm{H}_{\mathrm{g}}\right.$ and $\left.\mathrm{H}_{\mathrm{j}}\right) .{ }^{13} \mathrm{C}$ NMR $\left(100 \mathrm{MHz}, \mathrm{DMSO}-d_{6}\right): \delta 174.15(\mathrm{C}=\mathrm{O}), 169.03(\mathrm{C}=\mathrm{O}), 155.52(\mathrm{C}=\mathrm{O})$, $143.93(\mathrm{C}), 133.98(\mathrm{C}-\mathrm{Cl}), 132.50(\mathrm{C}-\mathrm{Cl}), 130.25\left(\mathrm{C}_{7}\right.$ and $\left.\mathrm{C}\right), 129.72\left(\mathrm{C}_{8}\right), 129.01\left(\mathrm{C}_{2}\right)$, $128.22\left(\mathrm{C}_{6}\right), 122.81\left(\mathrm{C}_{\mathrm{d}}\right), 122.18\left(\mathrm{C}_{4}\right), 122.08\left(\mathrm{C}_{5}\right), 116.05(\mathrm{CN}), 92.00\left(\mathrm{C}_{3}\right), 77.92(\mathrm{C}), 57.57$ $\left(\mathrm{C}_{\mathrm{b}}\right), 53.46\left(\mathrm{C}_{\mathrm{i}}\right), 49.15\left(\mathrm{C}_{\mathrm{e}}\right), 34.52\left(\mathrm{C}_{\mathrm{c}}\right), 30.34\left(\mathrm{C}_{\mathrm{h}}\right), 29.46\left(\mathrm{C}_{\mathrm{f}}\right), 28.21\left(3 \mathrm{C}_{\mathrm{j}}\right), 22.63\left(\mathrm{C}_{\mathrm{g}}\right), 18.23$ $\left(\mathrm{C}_{\mathrm{a}}\right)$. HRMS (ESI, $\mathrm{CH}_{3} \mathrm{CN}$ ): $\mathrm{m} / \mathrm{z}$ calculated for $\mathrm{C}_{28} \mathrm{H}_{33} \mathrm{Cl}_{2} \mathrm{~N}_{7} \mathrm{O}_{5}[\mathrm{M}+\mathrm{Na}]^{+} 640.1818, \mathrm{~m} / z$ found 640.1812 .

\subsubsection{Coupling protected amino acid derivative with the carboxylic acid derivative of fenpiclonil 9 (Fig. 2; compound 12)}

We next investigated the preparation of another compound without the 1,2,3-triazole ring as spacer group in order to compare the interaction between these amino acid conjugates and their respective carriers. Amide bonds play a major role in biological systems but also in a wide range of molecules like commercial drugs. ${ }^{38}$ The carboxylic acid moiety was first activated by an appropriate coupling reagent to form the $O$-acylisourea, a process allowing to convert the hydroxyl group of the acid into a good leaving group. The $O$-acylisourea intermediate was obtained from compound 9 previously described ${ }^{23}$ using the water soluble 1-(3-dimethylaminopropyl)-3-ethylcarbodiimide hydrochloride $(\mathrm{EDCl}) \quad 48,49$ and 4dimethylaminopyridine (DMAP) as catalyst. ${ }^{50}$ Then, this intermediate compound reacted in situ with the $N$ - $\alpha$-Boc- $L$-lysine derivative 6 to give the desired compound 12 which was 
easily extracted by an organic solvant, the urea derivative by-product being eliminated in the aqueous layer.

Experimental procedure for the synthesis of compound 12 (Fig. 3)

To a solution of compound 9 (3.10 g, $10.0 \mathrm{mmol}, 1$ equiv) in anhydrous dichloromethane $(30 \mathrm{~mL})$ cooled to $0^{\circ} \mathrm{C}$ was added $N$ - $\alpha$-Boc- $L$-lysine $(7.41 \mathrm{~g}, 30.0 \mathrm{mmol}, 3$ equiv), EDCl (5.96 g, $31.0 \mathrm{mmol}, 3.1$ equiv) and 4-DMAP (123 mg, $1.00 \mathrm{mmol}, 0.1$ equiv). The reaction mixture was purged with nitrogen through the septum and then stirred at $0^{\circ} \mathrm{C}$ for $2 \mathrm{~h}$. The mixture was allowed to reach room temperature and then stirred for $18 \mathrm{~h}$. Water was added and the organic layer was extracted twice with dichloromethane. The combined organic layers were washed with water, dried over $\mathrm{MgSO}_{4}$, filtered and concentrated under vacuum. The crude product was purified by silica gel column chromatography using pentane/ethyl acetate (5: 5) as eluent to obtain compound 12 as a pink powder ( $0.97 \mathrm{~g}, 18 \%$ yield).

2-(Tert-butoxycarbonylamino)-6-[2-(3-cyano-4-(2,3-dichlorophenyl)-1H-pyrrol-1yl)propanamido]hexanoic acid (12)

$\mathrm{Rf}=0.16$ (ethyl acetate/methanol: 9/1); Mp = 107-108 ${ }^{\circ} \mathrm{C} .{ }^{1} \mathrm{H}$ NMR (400 MHz, DMSO- $\left.d_{6}\right): \delta$ 12.52 (s, 1H, OH), $8.28\left(\mathrm{t}, 1 \mathrm{H},{ }^{3} J=5.5 \mathrm{~Hz}, \mathrm{NH}\right), 7.85\left(\mathrm{~d}, 1 \mathrm{H},{ }^{4} \mathrm{~J}=2.2 \mathrm{~Hz}, \mathrm{H}_{2}\right), 7.68(\mathrm{dd}, 1 \mathrm{H}$, $\left.{ }^{3} J=7.2 \mathrm{~Hz},{ }^{4} J=2.3 \mathrm{~Hz}, \mathrm{H}_{8}\right), 7.49-7.43\left(\mathrm{~m}, 2 \mathrm{H}, \mathrm{H}_{6}\right.$ and $\left.\mathrm{H}_{7}\right), 7.28\left(\mathrm{~d}, 1 \mathrm{H},{ }^{4} J=2.2 \mathrm{~Hz}, \mathrm{H}_{5}\right)$, $7.05(\mathrm{~s}, 1 \mathrm{H}, \mathrm{NH}), 4.90\left(\mathrm{q}, 1 \mathrm{H},{ }^{3} J=7.0 \mathrm{~Hz}, \mathrm{H}_{\mathrm{b}}\right), 3.88-3.83\left(\mathrm{~m}, 1 \mathrm{H}, \mathrm{H}_{\mathrm{g}}\right), 3.16-3.01\left(\mathrm{~m}, 2 \mathrm{H}, \mathrm{H}_{\mathrm{c}}\right)$, 1.74-1.56 (m, 5H, $\mathrm{H}_{\mathrm{a}}$ and $\left.\mathrm{H}_{\mathrm{f}}\right), 1.50-1.27\left(\mathrm{~m}, 13 \mathrm{H}, \mathrm{H}_{\mathrm{d}}, \mathrm{H}_{\mathrm{e}}\right.$ and $\left.\mathrm{H}_{\mathrm{h}}\right) .{ }^{13} \mathrm{C} \mathrm{NMR}(100 \mathrm{MHz}$, DMSO- $\left.d_{6}\right): \delta 174.23(\mathrm{C}=\mathrm{O}), 168.94(\mathrm{C}=\mathrm{O}), 155.59(\mathrm{C}=\mathrm{O}), 133.99(\mathrm{C}-\mathrm{Cl}), 132.47(\mathrm{C}-\mathrm{Cl})$, $130.26\left(\mathrm{C}_{7}\right), 130.24(\mathrm{C}), 129.71\left(\mathrm{C}_{8}\right), 128.95\left(\mathrm{C}_{2}\right), 128.22\left(\mathrm{C}_{6}\right), 122.14\left(\mathrm{C}_{5}\right), 122.01\left(\mathrm{C}_{4}\right)$, $116.05(\mathrm{CN}), 91.92\left(\mathrm{C}_{3}\right), 77.94(\mathrm{C}), 57.74\left(\mathrm{C}_{\mathrm{b}}\right), 53.36\left(\mathrm{C}_{\mathrm{g}}\right), 38.89\left(\mathrm{C}_{\mathrm{c}}\right), 30.69\left(\mathrm{C}_{\mathrm{f}}\right), 28.38\left(\mathrm{C}_{\mathrm{d}}\right)$, $28.19\left(3 \mathrm{C}_{\mathrm{h}}\right), 22.96\left(\mathrm{C}_{\mathrm{e}}\right), 18.33\left(\mathrm{C}_{\mathrm{a}}\right)$. HRMS (ESI, $\left.\mathrm{CH}_{3} \mathrm{CN}\right): \mathrm{m} / z$ calculated for $\mathrm{C}_{25} \mathrm{H}_{30} \mathrm{Cl}_{2} \mathrm{~N}_{4} \mathrm{O}_{5}$ $[\mathrm{M}+\mathrm{Na}]^{+} 559.1491, \mathrm{~m} / \mathrm{z}$ found 559.1492 .

\subsubsection{Deprotection of the amino acid moiety (Fig. 2; compounds $L-14, L-15, L-16$ )}

The last step consisted of the deprotection of the $\alpha$-aminoacid function of the compounds $\mathbf{1 1} \mathbf{R}_{\mathbf{2}}, \mathbf{1 1} \mathbf{R}_{\mathbf{3}}$ and $\mathbf{1 2}$. $^{51}$ The reaction was performed with trifluoroacetic acid in dichloromethane for $1 \mathrm{~h}$ at room temperature, permitting to remove the protecting $t$ butoxycarbonyl group of the amino function and led respectively to the final compounds $\mathbf{L}$ 14, L-15 and L-16 with 98\% yield.

Experimental procedure for the synthesis of compounds L-14, L-15, L-16 (Fig. 3)

The compound $\mathbf{1 1 R}_{\mathbf{2}}(1.77 \mathrm{~g}, 3.0 \mathrm{mmol})$ or $\mathbf{1 1 R _ { 3 }}(1.86 \mathrm{~g}, 3.0 \mathrm{mmol})$ or $\mathbf{1 2}(1.61 \mathrm{~g}, 3.0 \mathrm{mmol})$ was diluted in a mixture anhydrous dichloromethane - trifluoroacetic acid $(9.4 \mathrm{~mL}, 1: 1)$. The 
reaction mixture was purged with nitrogen through the septum and then stirred at room temperature for $1 \mathrm{~h}$ before being evaporated. The residue was co-evaporated with ethyl acetate, dried under vacuum to obtain respectively the compound L-14 as a pink powder (1.4 g, 98\% yield), L-15 as a beige powder (1.5 g, 98\% yield) or L-16 as a pink powder (1.44 g, 98\% yield).

2-Amino-4-(4-\{[2-(3-cyano-4-(2,3-dichlorophenyl)-1H-pyrrol-1-yl)propanamido]methyl $\}$ 1H-1,2,3-triazol-1-yl)butanoic acid (L-14)

$\mathrm{Rf}=0.12$ (ethyl acetate/methanol: $5 / 5) ; \mathrm{Mp}=174-175{ }^{\circ} \mathrm{C} .{ }^{1} \mathrm{H}$ NMR $\left(400 \mathrm{MHz}\right.$, DMSO- $\left.d_{6}\right): \delta$ $8.87\left(\mathrm{t}, 1 \mathrm{H},{ }^{3} J=5.3 \mathrm{~Hz}, \mathrm{NH}\right), 8.46\left(\mathrm{~s}, 3 \mathrm{H}, \mathrm{NH}_{2}\right.$ and $\left.\mathrm{OH}\right), 8.03\left(\mathrm{~s}, 1 \mathrm{H}, \mathrm{H}_{\mathrm{d}}\right), 7.87\left(\mathrm{~d}, 1 \mathrm{H},{ }^{4} J=\right.$ $\left.2.2 \mathrm{~Hz}, \mathrm{H}_{2}\right), 7.68\left(\mathrm{dd}, 1 \mathrm{H},{ }^{3} J=7.4 \mathrm{~Hz},{ }^{4} J=2.2 \mathrm{~Hz}, \mathrm{H}_{8}\right), 7.49-7.42\left(\mathrm{~m}, 2 \mathrm{H}, \mathrm{H}_{6}\right.$ and $\left.\mathrm{H}_{7}\right), 7.30$ $\left(\mathrm{d}, 1 \mathrm{H},{ }^{4} \mathrm{~J}=2.2 \mathrm{~Hz}, \mathrm{H}_{5}\right), 4.98\left(\mathrm{q}, 1 \mathrm{H},{ }^{3} J=7.0 \mathrm{~Hz}, \mathrm{H}_{\mathrm{b}}\right), 4.56\left(\mathrm{t}, 2 \mathrm{H},{ }^{3} J=7.5 \mathrm{~Hz}, \mathrm{H}_{\mathrm{e}}\right), 4.39$ (t, $\left.2 \mathrm{H},{ }^{3} J=5.3 \mathrm{~Hz}, \mathrm{H}_{\mathrm{c}}\right), 3.98-3.96\left(\mathrm{~m}, 1 \mathrm{H}, \mathrm{H}_{\mathrm{g}}\right), 2.46-2.26\left(\mathrm{~m}, 2 \mathrm{H}, \mathrm{H}_{\mathrm{f}}\right), 1.64\left(\mathrm{~d}, 3 \mathrm{H},{ }^{3} J=7.0 \mathrm{~Hz}\right.$, $\left.\mathrm{H}_{\mathrm{a}}\right) \cdot{ }^{13} \mathrm{C}$ NMR $\left(100 \mathrm{MHz}, \mathrm{DMSO}-d_{6}\right): \delta 170.25(\mathrm{C}=\mathrm{O}), 169.11(\mathrm{C}=\mathrm{O}), 144.23(\mathrm{C}), 133.96(\mathrm{C}-$ $\mathrm{Cl}), 132.49(\mathrm{C}-\mathrm{Cl}), 130.24\left(\mathrm{C}_{7}\right.$ and $\left.\mathrm{C}\right), 129.73\left(\mathrm{C}_{8}\right), 129.02\left(\mathrm{C}_{2}\right), 128.23\left(\mathrm{C}_{6}\right), 123.25\left(\mathrm{C}_{\mathrm{d}}\right)$, $122.16\left(\mathrm{C}_{4}\right), 122.09\left(\mathrm{C}_{5}\right), 116.05(\mathrm{CN}), 91.99\left(\mathrm{C}_{3}\right), 57.58\left(\mathrm{C}_{\mathrm{b}}\right), 49.67\left(\mathrm{C}_{\mathrm{g}}\right), 45.59\left(\mathrm{C}_{\mathrm{e}}\right), 34.45$ $\left(\mathrm{C}_{\mathrm{c}}\right), 30.73\left(\mathrm{C}_{\mathrm{f}}\right), 18.29\left(\mathrm{C}_{\mathrm{a}}\right)$. HRMS (ESI, $\left.\mathrm{CH}_{3} \mathrm{CN}\right): \mathrm{m} / z$ calculated for $\mathrm{C}_{21} \mathrm{H}_{21} \mathrm{Cl}_{2} \mathrm{~N}_{7} \mathrm{O}_{3}[\mathrm{M}+$ $\mathrm{Na}]^{+} 512.0981, \mathrm{~m} / \mathrm{z}$ found 512.0984 .

2-Amino-6-(4-\{[2-(3-cyano-4-(2,3-dichlorophenyl)-1H-pyrrol-1-yl)propanamido]methyl $\}$ 1H-1,2,3-triazol-1-yl)hexanoic acid (L-15)

$\mathrm{Rf}=0.14$ (ethyl acetate/methanol: $5 / 5) ; \mathrm{Mp}=129-130{ }^{\circ} \mathrm{C} .{ }^{1} \mathrm{H}$ NMR $\left(400 \mathrm{MHz}, \mathrm{DMSO}-d_{6}\right): \delta$ $8.86\left(\mathrm{t}, 1 \mathrm{H},{ }^{3} J=5.5 \mathrm{~Hz}, \mathrm{NH}\right), 8.28\left(\mathrm{~s}, 3 \mathrm{H}, \mathrm{NH}_{2}\right.$ and $\left.\mathrm{OH}\right), 7.97\left(\mathrm{~s}, 1 \mathrm{H}, \mathrm{H}_{\mathrm{d}}\right), 7.87\left(\mathrm{~d}, 1 \mathrm{H},{ }^{4} J=\right.$ $\left.2.3 \mathrm{~Hz}, \mathrm{H}_{2}\right), 7.68\left(\mathrm{dd}, 1 \mathrm{H},{ }^{3} J=7.4 \mathrm{~Hz},{ }^{4} J=2.2 \mathrm{~Hz}, \mathrm{H}_{8}\right), 7.49-7.42\left(\mathrm{~m}, 2 \mathrm{H}, \mathrm{H}_{6}\right.$ and $\left.\mathrm{H}_{7}\right), 7.30$ $\left(\mathrm{d}, 1 \mathrm{H},{ }^{4} J=2.3 \mathrm{~Hz}, \mathrm{H}_{5}\right), 4.99\left(\mathrm{q}, 1 \mathrm{H},{ }^{3} J=7.0 \mathrm{~Hz}, \mathrm{H}_{\mathrm{b}}\right), 4.38-4.34\left(\mathrm{~m}, 4 \mathrm{H}, \mathrm{H}_{\mathrm{c}}\right.$ and $\left.\mathrm{H}_{\mathrm{e}}\right), 3.95-$ $3.92\left(\mathrm{~m}, 1 \mathrm{H}, \mathrm{H}_{\mathrm{i}}\right), 1.88-1.64\left(\mathrm{~m}, 4 \mathrm{H}, \mathrm{H}_{\mathrm{f}}\right.$ and $\left.\mathrm{H}_{\mathrm{h}}\right), 1.49\left(\mathrm{~d}, 3 \mathrm{H},{ }^{3} J=7.0 \mathrm{~Hz}, \mathrm{H}_{\mathrm{a}}\right), 1.34-1.24(\mathrm{~m}$, 2H, $\left.\mathrm{H}_{\mathrm{g}}\right) \cdot{ }^{13} \mathrm{C}$ NMR (100 MHz, DMSO-d $\left.)_{6}\right): \delta 172.09(\mathrm{C}=\mathrm{O}), 170.12(\mathrm{C}=\mathrm{O}), 144.97(\mathrm{C}), 134.79$ (C-Cl), $133.32(\mathrm{C}-\mathrm{Cl}), 131.06\left(\mathrm{C}_{7}\right.$ and $\left.\mathrm{C}\right), 130.55\left(\mathrm{C}_{8}\right), 129.81\left(\mathrm{C}_{2}\right), 129.03\left(\mathrm{C}_{6}\right), 123.53\left(\mathrm{C}_{\mathrm{d}}\right)$, $122.94\left(\mathrm{C}_{4}\right), 122.84\left(\mathrm{C}_{5}\right), 116.77(\mathrm{CN}), 92.01\left(\mathrm{C}_{3}\right), 57.60\left(\mathrm{C}_{\mathrm{b}}\right), 51.76\left(\mathrm{C}_{\mathrm{i}}\right), 48.97\left(\mathrm{C}_{\mathrm{e}}\right), 34.50$ $\left(\mathrm{C}_{\mathrm{c}}\right), 29.43\left(\mathrm{C}_{\mathrm{h}}\right), 29.28\left(\mathrm{C}_{\mathrm{f}}\right), 21.49\left(\mathrm{C}_{\mathrm{g}}\right), 18.24\left(\mathrm{C}_{\mathrm{a}}\right)$. HRMS (ESI, $\left.\mathrm{CH}_{3} \mathrm{CN}\right): m / z$ calculated for $\mathrm{C}_{23} \mathrm{H}_{25} \mathrm{Cl}_{2} \mathrm{~N}_{7} \mathrm{O}_{3}[\mathrm{M}+\mathrm{Na}]^{+}$518.1474, $\mathrm{m} / z$ found 518.1483 .

2-Amino-6-[2-(3-cyano-4-(2,3-dichlorophenyl)-1H-pyrrol-1-yl)propanamido]hexanoic acid (L-16)

$\mathrm{Rf}=0.14$ (ethyl acetate/methanol: $5 / 5) ; \mathrm{Mp}=112-113{ }^{\circ} \mathrm{C} .{ }^{1} \mathrm{H}$ NMR $\left(400 \mathrm{MHz}, \mathrm{DMSO}-d_{6}\right): \delta$ $8.35\left(\mathrm{t}, 1 \mathrm{H},{ }^{3} J=5.5 \mathrm{~Hz}, \mathrm{NH}\right), 8.27\left(\mathrm{~s}, 3 \mathrm{H}, \mathrm{NH}_{2}\right.$ and $\left.\mathrm{OH}\right), 7.85\left(\mathrm{~d}, 1 \mathrm{H},{ }^{4} J=2.2 \mathrm{~Hz}, \mathrm{H}_{2}\right), 7.68$ 
(dd, $\left.1 \mathrm{H},{ }^{3} J=7.4 \mathrm{~Hz},{ }^{4} J=2.2 \mathrm{~Hz}, \mathrm{H}_{8}\right), 7.49-7.43\left(\mathrm{~m}, 2 \mathrm{H}, \mathrm{H}_{6}\right.$ and $\left.\mathrm{H}_{7}\right), 7.28\left(\mathrm{~d}, 1 \mathrm{H},{ }^{4} J=2.2 \mathrm{~Hz}\right.$, $\left.\mathrm{H}_{5}\right), 4.90\left(\mathrm{q}, 1 \mathrm{H},{ }^{3} J=7.0 \mathrm{~Hz}, \mathrm{H}_{\mathrm{b}}\right), 3.92-3.91\left(\mathrm{~m}, 1 \mathrm{H}, \mathrm{H}_{\mathrm{g}}\right), 3.17-3.05\left(\mathrm{~m}, 2 \mathrm{H}, \mathrm{H}_{\mathrm{c}}\right), 1.85-1.71$ $\left(\mathrm{m}, 2 \mathrm{H}, \mathrm{H}_{\mathrm{f}}\right), 1.63\left(\mathrm{~d}, 3 \mathrm{H},{ }^{3} \mathrm{~J}=7.0 \mathrm{~Hz}, \mathrm{H}_{\mathrm{a}}\right), 1.49-1.26\left(\mathrm{~m}, 4 \mathrm{H}, \mathrm{H}_{\mathrm{d}}\right.$ and $\left.\mathrm{H}_{\mathrm{e}}\right) .{ }^{13} \mathrm{C} \mathrm{NMR}(100 \mathrm{MHz}$, DMSO- $\left.d_{6}\right)$ : $\delta 171.09(\mathrm{C}=\mathrm{O}), 169.02(\mathrm{C}=\mathrm{O}), 133.98(\mathrm{C}-\mathrm{Cl}), 132.49(\mathrm{C}-\mathrm{Cl}), 130.26\left(\mathrm{C}_{7}\right.$ and $\left.\mathrm{C}\right)$, $129.74\left(\mathrm{C}_{8}\right), 128.94\left(\mathrm{C}_{2}\right), 128.25\left(\mathrm{C}_{6}\right), 122.15\left(\mathrm{C}_{4}\right), 122.03\left(\mathrm{C}_{5}\right), 116.06(\mathrm{CN}), 91.94\left(\mathrm{C}_{3}\right)$, $57.74\left(\mathrm{C}_{\mathrm{b}}\right), 51.85\left(\mathrm{C}_{\mathrm{g}}\right), 38.89\left(\mathrm{C}_{\mathrm{c}}\right), 29.62\left(\mathrm{C}_{\mathrm{f}}\right), 28.29\left(\mathrm{C}_{\mathrm{d}}\right), 21.70\left(\mathrm{C}_{\mathrm{e}}\right), 18.34\left(\mathrm{C}_{\mathrm{a}}\right)$. HRMS (ESI, $\left.\mathrm{CH}_{3} \mathrm{CN}\right): m / z$ calculated for $\mathrm{C}_{20} \mathrm{H}_{22} \mathrm{Cl}_{2} \mathrm{~N}_{4} \mathrm{O}_{3}[\mathrm{M}+\mathrm{Na}]^{+} 459.0967, m / z$ found 459.0986 .

\subsection{Comparison of the phloem mobility of compounds L-13, L-14, L-15 and L-16 using the Ricinus model}

The permeability of plant plasma membrane is one of the key determinant of pesticide phloem systemicity, because any pesticides that can be transported in the vascular system must cross the plasma membrane before entering the symplast. ${ }^{52}$ The classical approach to improve xenobiotic uptake can be achieved through optimizing the physicochemical properties to increase passive membrane penetration. ${ }^{53}$ Chemical descriptors and physicochemical properties of our conjugates were calculated and listed in Table 2. Lipinski's rule of five (Ro5) and Veber's rule which have been proven as effective approaches first for drug bioavailability ${ }^{54,55}$ and then for agrochemicals uptake, ${ }^{56,57}$ were used to predict plant membrane permeability of L-13, L-14, L-15 and L-16. It should be noted that passive diffusion models are not applicable to active carrier-mediated molecules. ${ }^{58}$ In addition, they must be supplemented by other models $53,59,60$ to explain phloem systemicity or nonsystemicity. For instance, fenpiclonil which is predicted to diffuse through membranes (Table 2 ) is not phloem mobile in agreement with the Kleier's map. ${ }^{23}$

To determine the influence and contribution of the spacer arm structure on phloem mobility, the three new conjugates were assessed in the Ricinus model under the same experimental conditions. The cotyledons of Ricinus seedlings were incubated with tested conjugates at 100 $\mu \mathrm{M}$ concentration at $\mathrm{pH} 5.0$ which is close to the $\mathrm{pH}$ value of the cotyledon tissue apoplast under our experimental conditions. Our previous study revealed that the concentration of conjugate L-13 in phloem sap reached a plateau at about $12 \mu \mathrm{M}$ after $5 \mathrm{~h}$ incubation. ${ }^{24}$ In the present study, the concentration of conjugates L-15 and L-16 increased linearly up to 5-6 h to reach about $20 \mu \mathrm{M}$ under our experimental conditions (Fig. 4). The time-course concentration of conjugate L-14 (which violates the Veber's rule, Table 2) exhibited the same pattern but showed the best systemicity (Fig. 4). At time $6 \mathrm{~h}$, its concentration in the phloem sap (about 
$30 \mu \mathrm{M}$ ) was 2.5 times higher than these of conjugate $\mathbf{L - 1 3}$ and a glycinergic-fipronil conjugate recently synthesized. ${ }^{19}$

A comparison between conjugates L-13, L-14 and L-15 which contain the same triazole spacer indicated that reducing the chain of the $L$-amino acid increased phloem mobility, notably by removing the amido group (Fig. 4). In contrast, comparison between conjugate L15 (which violates both Lipinski's and Veber's rules, Table 2) and conjugate L-16 (which is predicted to diffuse easily through membranes, Table 2) showed that removing the triazole ring did not influence positively phloem mobility (Fig. 4). In other words, addition of a triazole spacer did not affect the systemicity of our compounds. Finally, our data are in agreement with the paradigm that passive diffusion models are not suitable to predict transport mediated by active carrier systems. ${ }^{58}$

\subsection{Comparison of phloem mobility of conjugate L-14 and acidic derivatives of fenpiclonil in the physiological pH range of mature leaf apoplast}

For about three decades, fluorescence methods have been used to measure the apoplast $\mathrm{pH}$ of the leaf tissues of a large number of plant species, gymnosperms included. The data indicate that it ranges from 5.0 to 6.6 according to species, cell types and environmental conditions. ${ }^{61-}$ ${ }^{67} \mathrm{pH}$ gradients of 0.5-1.0 unit exist between the vein apoplast and the mesophyll apoplast. For instance, in Helianthus annus the apoplastic pH values are about 5.6 and 6.2 in the vein area and the mesophyll respectively. ${ }^{64}$ The most acidic $\mathrm{pH}$ values are found in Vicia faba and varies from $\mathrm{pH} 5.0$ in light period to $\mathrm{pH} 5.4$ in dark period. This oscillation is possibly due to changes in the activity of the plasmamembrane H+-ATPase ${ }^{64}$ which is highly expressed in the phloem tissue of this apoplastic loader. ${ }^{68}$ There are two strategies in developing pesticides with an efficient phloem systemicity, initially the ion-trap mechanism ${ }^{53,59,69}$ and recently, the

carrier-mediated process. ${ }^{16}$ Using the ion-trap-mechanism, fenpiclonil was modified by adding carboxylic acid group in previous works. ${ }^{22,23}$ The concentration of these acidic derivatives in the phloem sap was closely correlated to the percentage of their undissociated form in the external incubation solution at various biological $\mathrm{pH}$ values and droped from $\mathrm{pH}$ 5.0 to $\mathrm{pH}$ 6.0. For instance, the concentration factor of compound 9 (Table 2) was $0.3,0.04$ and below 0.001 at $\mathrm{pH}$ 5.0, 5.5 and 6.0 respectively (Fig. 5B). By contrast, the concentration factor of conjugate L-14 did not exhibit significant change from $\mathrm{pH} 5.0$ to 6.0 (Fig. 5A). Then, it decreased by about 3-4 times at pHs 7.0 and 8.0. This $\mathrm{pH}$ dependence was similar to active uptake of neutral amino acids which are manipulated by proton-amino acid symporters. ${ }^{70,71}$ There was a residual uptake at neutral and slightly alkaline $\mathrm{pH}$ values (Fig. 5A) as it is 
the case for neutral amino acid uptake by leaf tissues. ${ }^{72}$ The different phloem mobility behaviors of fenpiclonil acidic derivatives and compound L-14 indicate that carrier-mediated processes can be more efficient for confering systemicity to phenylpyrroles taking into account the $\mathrm{pH}$ values of the leaf tissue apoplast mentionned above.

\section{CONCLUSION}

In this study, three $L$-amino acid conjugates of fenpiclonil exhibiting a free $L$ - $\alpha$-amino acid function were successfully prepared with different length of spacer arm. The selected spacer structure incorporated a triazole ring for two conjugates. The phloem mobility of these conjugates was investigated using the Ricinus model. The results showed that the conjugates were manipulated by a $\mathrm{pH}$-dependent transporter system recognizing the $L$ - $\alpha$-amino acid function and translocated at distance in the phloem. Despite the steric hindrance due to these large chlorinated conjugates, i.e. xenobiotics much larger than natural substrates and exhibiting different physico-chemical properties, it is possible to clearly improve their phloem mobility by reducing the length of the $L$-amino acid chain. While the phloem systemicity of acidic derivatives of fenpiclonil was limited to $\mathrm{pH}$ values near 5.0, the carrier-mediated conjugates can be transported in the whole range of foliar apoplast $\mathrm{pH}$ values (from 5.0 to 6.5) measured in Angiosperms and Gymnosperms (Fig. 6).

Click chemistry is a practical and reliable approach in conjugation reaction. ${ }^{73}$ Introduction of a triazole ring in the spacer of our conjugates did not display any negative effect on phloem mobility. In addition, no parent compound was detected in phloem sap after the treatment of all four conjugates, indicating that all selected spacer arms were non-degradable in the companion cell-sieve cell complex. However, as a successful prodrug strategy, the active ingredient should be released from the prodrug to exert its biological action. Therefore, in addition of studying the distribution pattern of conjugates within the whole plant as indicated before, ${ }^{24}$ it will be interesting to focus the investigations on degradable spacer group in order to release the active ingredient. For example, $\mathrm{pH}$-sensitive linkage can be used based on the pH difference between the leaf apoplast and symplast. ${ }^{74}$

\section{ACKNOWLEDGMENTS}

The authors are grateful to FranceAgriMer, InterLoire and Jas Hennessy \& Co. for their financial support of this work. This work was also supported by China Scholarship Council (Hanxiang $\mathrm{Wu}$ grant for his $\mathrm{PhD}$ ). We would like to thank Emilie Dugaro for her kind 
collaboration in organic synthesis and Dr Rémi Lemoine for his help to improve the manuscript.

\section{REFERENCES}

1. Chandrasekaran J, Brumin M, Wolf D, Leibman D, Klap C, Pearlsman M, Sherman A, Arazi T and Gal-On A, Development of broad virus resistance in non-transgenic cucumber using CRISPR/Cas9 technology. Mol Plant Pathol 17: 1140-1153 (2016).

2. Dedryver CA, Le Ralec A and Fabre F, The conflicting relationships between aphids and men: A review of aphid damage and control strategies. C R Biol 333: 539-553 (2010).

3. Dogimont C, Bendahmane A, Chovelon V and Boissot N, Host plant resistance to aphids in cultivated crops: Genetic and molecular bases, and interactions with aphid populations. C R Biol 333: 566-573 (2010).

4. Han HK and Amidon GL, Targeted prodrug design to optimize drug delivery. AAPS PharmSci 2: E6 (2000).

5. Jeschke P, Propesticides and their use as agrochemicals. Pest Manage Sci 72: 210225 (2016).

6. Smith PH, Chamberlain K, Sugars JM and Bromilow RH, Fungicidal activity of $N$-(2cyano-2-methoximinoacetyl)amino acids and their derivatives. Pestic Sci 44: 219-224 (1995).

7. del Amo EM, Urtti A and Yliperttula M, Pharmacokinetic role of L-type amino acid transporters LAT1 and LAT2. Eur J Pharm Sci 35: 161-174 (2008).

8. Dobson PD and Kell DB, Carrier-mediated cellular uptake of pharmaceutical drugs: an exception or the rule? Nat Rev Drug Discov 7: 205-220 (2008).

9. Fan W, Wu Y, Li XK, Yao N, Li X, Yu YG and Hai L, Design, synthesis and biological evaluation of brain-specific glucosyl thiamine disulfide prodrugs of naproxen. Eur J Med Chem 46: 3651-3661 (2011).

10. Janzer M, Larbig G, Kubelbeck A, Wischnjow A, Haberkorn U and Mier W, Drug conjugation affects pharmacokinetics and specificity of kidney-targeted peptide carriers. Bioconj Chem 27: 2441-2449 (2016).

11. Peura L, Malmioja K, Laine K, Leppanen J, Gynther M, Isotalo A and Rautio J, Large amino acid transporter 1 (LAT1) prodrugs of valproic acid: new prodrug design ideas for central nervous system delivery. Mol Pharm 8: 1857-1866 (2011).

12. Peura L, Malmioja K, Huttunen K, Leppanen J, Hamalainen M, Forsberg MM, Rautio $\mathrm{J}$ and Laine K, Design, synthesis and brain uptake of LAT1-targeted amino acid prodrugs of dopamine. Pharm Res 30: 2523-2537 (2013).

13. Sugano K, Kansy M, Artursson P, Avdeef A, Bendels S, Di L, Ecker GF, Faller B, Fischer H, Gerebtzoff G, Lennernaes H and Senner F, Coexistence of passive and carrier-mediated processes in drug transport. Nat Rev Drug Discov 9: 597-614 (2010).

14. Sugawara M, Huang W, Fei YJ, Leibach FH, Ganapathy V and Ganapathy ME, Transport of valganciclovir, a ganciclovir prodrug, via peptide transporters PEPT1 and PEPT2. J Pharm Sci 89: $781-789$ (2000). 
15. Zawilska JB, Wojcieszak J and Olejniczak AB, Prodrugs: A challenge for the drug development. Pharmacol Rep 65: 1-14 (2013).

16. Chollet JF, Deletage C, Faucher M, Miginiac L and Bonnemain JL, Synthesis and structure-activity relationships of some pesticides with an alpha-amino acid function. Biochim Biophys Acta 1336: 331-341 (1997).

17. Yang $\mathrm{W}, \mathrm{Wu} \mathrm{HX}, \mathrm{Xu} \mathrm{HH}, \mathrm{Hu} \mathrm{AL}$ and Lu ML, Synthesis of glucose-fipronil conjugate and its phloem mobility. J Agric Food Chem 59: 12534-12542 (2011).

18. Wu HX, Yang W, Zhang ZX, Huang T, Yao GK and Xu HH, Uptake and phloem transport of glucose-fipronil conjugate in Ricinus communis involve a carrier-mediated mechanism. J Agric Food Chem 60: 6088-6094 (2012).

19. Xie Y, Zhao JL, Wang CW, Yu AX, Liu N, Chen L, Lin F and Xu HH, GlycinergicFipronil uptake is mediated by an amino acid carrier system and induces the expression of amino acid transporter genes in Ricinus communis seedlings. J Agric Food Chem 64: 3810-3818 (2016).

20. Deletage-Grandon C, Chollet JF, Faucher M, Rocher F, Komor E and Bonnemain JL, Carrier-mediated uptake and phloem systemy of a 350-Dalton chlorinated xenobiotic with an alpha-amino acid function. Plant Physiol 125: 1620-1632 (2001).

21. Tjamos EC. Problems and prospects in controlling Verticillium wilt. In Vascular Wilt Diseases of Plants, ed. by E. C. Tjamos CHB. Springer Berlin Heidelberg, pp. 441456 (1989).

22. Chollet JF, Rocher F, Jousse C, Deletage-Grandon C, Bashiardes G and Bonnemain JL, Synthesis and phloem mobility of acidic derivatives of the fungicide fenpiclonil. Pest Manage Sci 60: 1063-1072 (2004).

23. Chollet JF, Rocher F, Jousse C, Deletage-Grandon C, Bashiardes G and Bonnemain JL, Acidic derivatives of the fungicide fenpiclonil: effect of adding a methyl group to the N-substituted chain on systemicity and fungicidal activity. Pest Manage Sci 61: 377-382 (2005).

24. Wu H, Marhadour S, Lei Z-W, Yang W, Marivingt-Mounir C, Bonnemain J-L and Chollet J-F, Vectorization of agrochemicals: amino acid carriers are more efficient than sugar carriers to translocate phenylpyrrole conjugates in the Ricinus system. Environ Sci Pollut Res Int: In press, DOI 10.1007/s11356-11016-18107-x (2016).

25. Brik A, Alexandratos J, Lin YC, Elder JH, Olson AJ, Wlodawer A, Goodsell DS and Wong $\mathrm{CH}, 1,2,3$-triazole as a peptide surrogate in the rapid synthesis of HIV-1 protease inhibitors. ChemBioChem 6: 1167-+ (2005).

26. Bock VD, Speijer D, Hiemstra H and van Maarseveen JH, 1,2,3-Triazoles as peptide bond isosteres: synthesis and biological evaluation of cyclotetrapeptide mimics. Org Biomol Chem 5: 971-975 (2007).

27. Valverde IE, Bauman A, Kluba CA, Vomstein S, Walter MA and Mindt TL, 1,2,3Triazoles as amide bond mimics: triazole scan yields protease-resistant peptidomimetics for tumor targeting. Angewandte Chemie-International Edition 52: 8957-8960 (2013).

28. Hein CD, Liu XM and Wang D, Click chemistry, a powerful tool for pharmaceutical sciences. Pharm Res 25: 2216-2230 (2008). 
29. Kallarackal J, Orlich G, Schobert C and Komor E, Sucrose transport into the phloem of Ricinus communis L seedlings as measured by the analysis of sieve-tube sap. Planta 177: 327-335 (1989).

30. Robinson SP and Beevers H, Amino acid transport in germinating castor bean seedlings. Plant Physiol 68: 560-566 (1981).

31. Komor E, Rotter M and Tanner W, Proton cotransport system in a higher plant sucrose transport in Ricinus communis. Plant Sci Lett 9: 153-162 (1977).

32. Rocher F, Chollet JF, Jousse C and Bonnemain JL, Salicylic acid, an ambimobile molecule exhibiting a high ability to accumulate in the phloem. Plant Physiol 141: 1684-1693 (2006).

33. Rocher F, Roblin G and Chollet J-F, Modifications of the chemical structure of phenolics differentially affect physiological activities in pulvinar cells of Mimosa pudica L. II. Influence of various molecular properties in relation to membrane transport. Environ Sci Pollut Res Int: In press, DOI 10.1007/s11356-11016-16048-z (2016).

34. Kolb HC, Finn MG and Sharpless KB, Click chemistry: diverse chemical function from a few good reactions. Angew Chem Int Ed 40: 2004-2021 (2001).

35. Leyden R and Murphy PV, Glycotriazolophane synthesis via click chemistry. Synlett: 1949-1950 (2009).

36. Meldal $\mathrm{M}$ and Tornoe $\mathrm{CW}, \mathrm{Cu}$-catalyzed azide-alkyne cycloaddition. Chem Rev 108: 2952-3015 (2008).

37. Sheehan JC, Boshart GL and Cruickshank PA, Convenient synthesis of water-soluble carbodiimides. J Org Chem 26: 2525-2528 (1961).

38. Valeur E and Bradley M, Amide bond formation: beyond the myth of coupling reagents. Chem Soc Rev 38: 606-631 (2009).

39. Sheehan JC and Hess GP, A new method of forming peptide bonds. J Am Chem Soc 77: 1067-1068 (1955).

40. Totaro KA, Liao XL, Bhattacharya K, Finneman JI, Sperry JB, Massa MA, Thorn J, Ho SV and Pentelute BL, Systematic investigation of EDC/sNHS-mediated bioconjugation reactions for carboxylated peptide substrates. Bioconj Chem 27: 9941004 (2016).

41. Cavender CJ and Shiner VJ, Trifluoromethanesulfonyl azide - its reaction with alkyl amines to form alkyl azides. J Org Chem 37: 3567-3569 (1972).

42. Das M, Bandyopadhyay D, Mishra D, Datir S, Dhak P, Jain S, Maiti TK, Basak A and Pramanik P, "Clickable", trifunctional magnetite nanoparticles and their chemoselective biofunctionalization. Bioconj Chem 22: 1181-1193 (2011).

43. Khoee $\mathrm{S}$ and Abedini N, One-pot synthesis of amphiphilic nanogels from vinylated SPIONs/HEMA/PEG via a combination of click chemistry and surfactant-free emulsion photopolymerization: Unveiling of the protein-nanoparticle interactions. Polymer 55: 5635-5647 (2014).

44. Titz A, Radic Z, Schwardt O and Ernst B, A safe and convenient method for the preparation of triflyl azide, and its use in diazo transfer reactions to primary amines. Tetrahedron Lett 47: 2383-2385 (2006). 
45. Link AJ, Vink MKS and Tirrell DA, Presentation and detection of azide functionality in bacterial cell surface proteins. J Am Chem Soc 126: 10598-10602 (2004).

46. Huisgen R, Knorr R, Mobius L and Szeimies G, 1.3-Dipolare cycloadditionen. 23. Einige beobachtungen zur addition organischer azide an cc-dreifachbindungen. Chem Ber Recl 98: 4014-4021 (1965).

47. Rostovtsev VV, Green LG, Fokin VV and Sharpless KB, A stepwise Huisgen cycloaddition process: Copper(I)-catalyzed regioselective "ligation" of azides and terminal alkynes. Angew Chem Int Ed 41: 2596-2599 (2002).

48. Han SY and Kim YA, Recent development of peptide coupling reagents in organic synthesis. Tetrahedron 60: 2447-2467 (2004).

49. Nakajima $\mathrm{N}$ and Ikada $\mathrm{Y}$, Mechanism of amide formation by carbodiimide for bioconjugation in aqueous-media. Bioconj Chem 6: 123-130 (1995).

50. Montalbetti $\mathrm{C}$ and Falque V, Amide bond formation and peptide coupling. Tetrahedron 61: 10827-10852 (2005).

51. Siebum AHG, Tsang RKF, van der Steen R, Raap J and Lugtenburg J, Synthesis of (epsilon-C-13-,epsilon N-15)-enriched L-lysine - Establishing schemes for the preparation of all possible C-13 and N-15 isotopomers of L-lysine, L-ornithine, and Lproline. Eur J Org Chem: 4391-4396 (2004).

52. Sicbaldi F, Sacchi GA, Trevisan M and DelRe AAM, Root uptake and xylem translocation of pesticides from different chemical classes. Pestic Sci 50: 111-119 (1997).

53. Kleier DA and Hsu FC, Phloem mobility of xenobiotics. 7.The design of phloem systemic pesticides. Weed Sci 44: 749-756 (1996).

54. Lipinski CA, Lombardo F, Dominy BW and Feeney PJ, Experimental and computational approaches to estimate solubility and permeability in drug discovery and development settings. Adv Drug Del Rev 64: 4-17 (2012).

55. Veber DF, Johnson SR, Cheng HY, Smith BR, Ward KW and Kopple KD, Molecular properties that influence the oral bioavailability of drug candidates. J Med Chem 45 : 2615-2623 (2002).

56. Tice CM, Selecting the right compounds for screening: does Lipinski's Rule of 5 for pharmaceuticals apply to agrochemicals? Pest Manage Sci 57: 3-16 (2001).

57. Avram S, Funar-Timofei S, Borota A, Chennamaneni SR, Manchala AK and Muresan $\mathrm{S}$, Quantitative estimation of pesticide-likeness for agrochemical discovery. $J$ Cheminform 6: 11 (2014).

58. Rocher F, Chollet JF, Legros S, Jousse C, Lemoine R, Faucher M, Bush DR and Bonnemain JL, Salicylic acid transport in Ricinus communis involves a $\mathrm{pH}$-dependent carrier system in addition to diffusion. Plant Physiol 150: 2081-2091 (2009).

59. Bromilow RH, Chamberlain K and Evans AA, Physicochemical aspects of phloem translocation of herbicides. Weed Sci 38: 305-314 (1990).

60. Satchivi NM, Stoller EW, Wax LM and Briskin DP, A nonlinear, dynamic, simulation model for transport, and whole plant allocation of systemic xenobiotics following foliar application. IV: Physicochemical properties requirements for optimum absorption and translocation. Pestic Biochem Physiol 84: 83-97 (2006). 
61. Husted S and Schjoerring JK, Apoplastic $\mathrm{pH}$ and ammonium concentration in leaves of Brassica napus L. Plant Physiol 109: 1453-1460 (1995).

62. López-Millán AF, Morales F, Abadia A and Abadia J, Effects of iron deficiency on the composition of the leaf apoplastic fluid and xylem sap in sugar beet. Implications for iron and carbon transport. Plant Physiol 124: 873-884 (2000).

63. Loubet B, Milford C, Hill PW, Tang YS, Cellier P and Sutton MA, Seasonal variability of apoplastic $\mathrm{NH} 4+$ and $\mathrm{pH}$ in an intensively managed grassland. Plant Soil 238: $97-110$ (2002).

64. Mühling KH and Läuchli A, Light-induced $\mathrm{pH}$ and $\mathrm{K}+$ changes in the apoplast of intact leaves. Planta 212: 9-15 (2000).

65. Mühling KH, Plieth C, Hansen UP and Sattelmacher B, Apoplastic pH of intact leaves of Vicia faba as influenced by light. J Exp Bot 46: 377-382 (1995).

66. Pfanz H and Dietz KJ, A fluorescence method for the determination of the apoplastic proton concentration in intact leaf tissues. J Plant Physiol 129: 41-48 (1987).

67. Pitann B, Schubert S and Mühling KH, Decline in leaf growth under salt stress is due to an inhibition of $\mathrm{H}+$-pumping activity and increase in apoplastic $\mathrm{pH}$ of maize leaves. J Plant Nutr Soil Sci 172: 535-543 (2009).

68. Bouché-Pillon S, Fleurat-Lessard P, Fromont JC, Serrano R and Bonnemain JL, Immunolocalization of the plasma membrane H+-ATPase in minor veins of Vicia faba in relation to phloem loading. Plant Physiol 105: 691-697 (1994).

69. Delrot $\mathrm{S}$ and Bonnemain J. Absorption et migration des herbicides. In Les herbicides, mode d'action et principes d'utilisation, ed. by Scala R. éditions INRA: Paris, France, pp. 51-77 (1991).

70. Ortiz-Lopez A, Chang HC and Bush DR, Amino acid transporters in plants. Biochim Biophys Acta 1465: 275-280 (2000).

71. Tegeder M and Ward JM, Molecular evolution of plant AAP and LHT amino acid transporters. Front Plant Sci 3: 11 (2012).

72. Despeghel JP and Delrot S, Energetics of amino acid uptake by Vicia faba leaf tissues. Plant Physiol 71: 1-6 (1983).

73. Kolb HC and Sharpless KB, The growing impact of click chemistry on drug discovery. Drug Discov Today 8: 1128-1137 (2003).

74. Gillies ER, Goodwin AP and Frechet JMJ, Acetals as pH-sensitive linkages for drug delivery. Bioconj Chem 15: 1254-1263 (2004). 

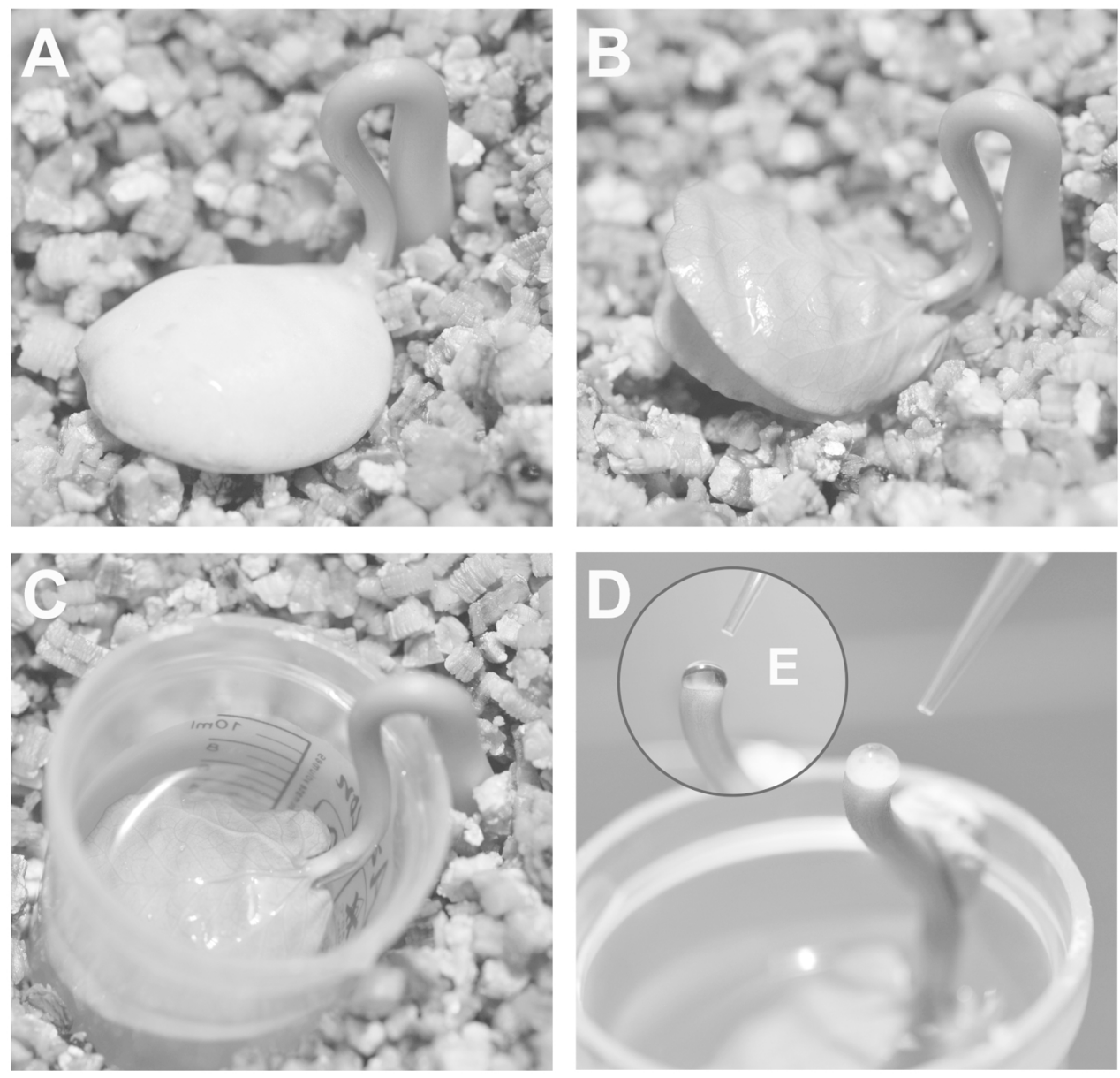

Figure 1. The Ricinus model. (A) The ricinus seedlings 6 days after sowing. (B) The endosperm was careffully removed. (C) Cotyledons were immersed in a buffered solution containing the tested product. (D)(E) After cutting the hypocotyl, phloem sap accumulated on the cross section and then was collected with a micropipette. See also Fig. 1 from Kallarackal et al (28). 
- First step (only when spacer includes 1,2,3-triazole ring): obtention of azido derivatives $N_{3}-R_{x}$ from protected amino acids

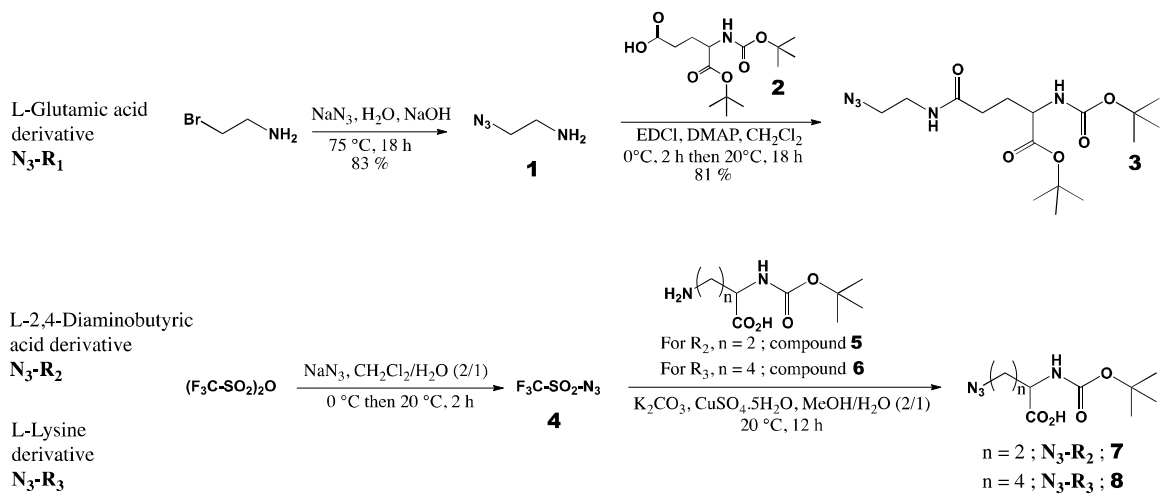

- Second step: coupling azido derivatives $\mathbf{N}_{3}-\mathbf{R}_{\mathbf{x}}$ and a propargyl derivative of fenpiclonil by click chemistry (when spacer includes 1,2,3-triazole ring) or protected amino acid derivative 6 with the carboxylic acid derivative of fenpiclonil 9

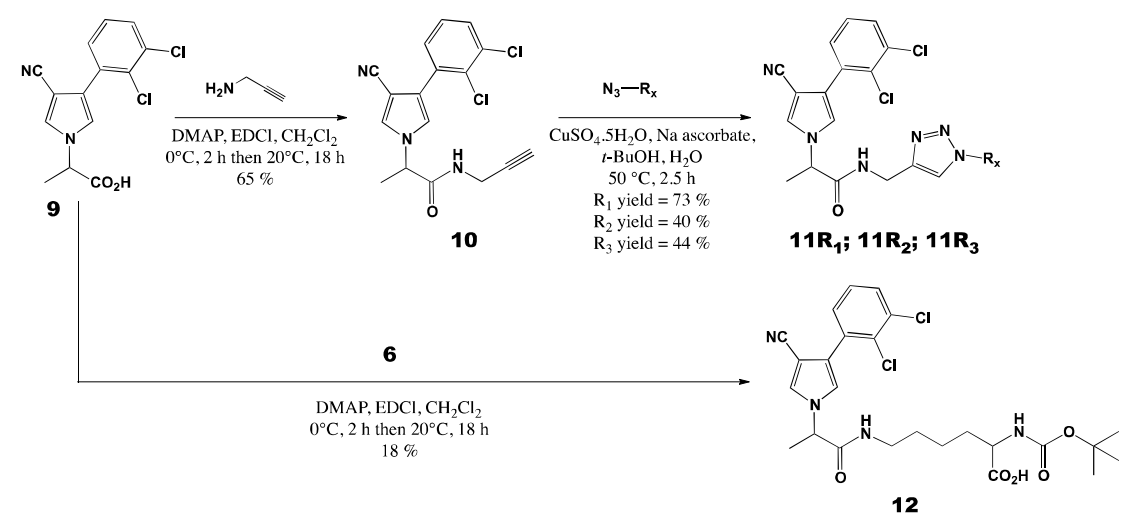

- Third step: deprotection of the amino acid moiety

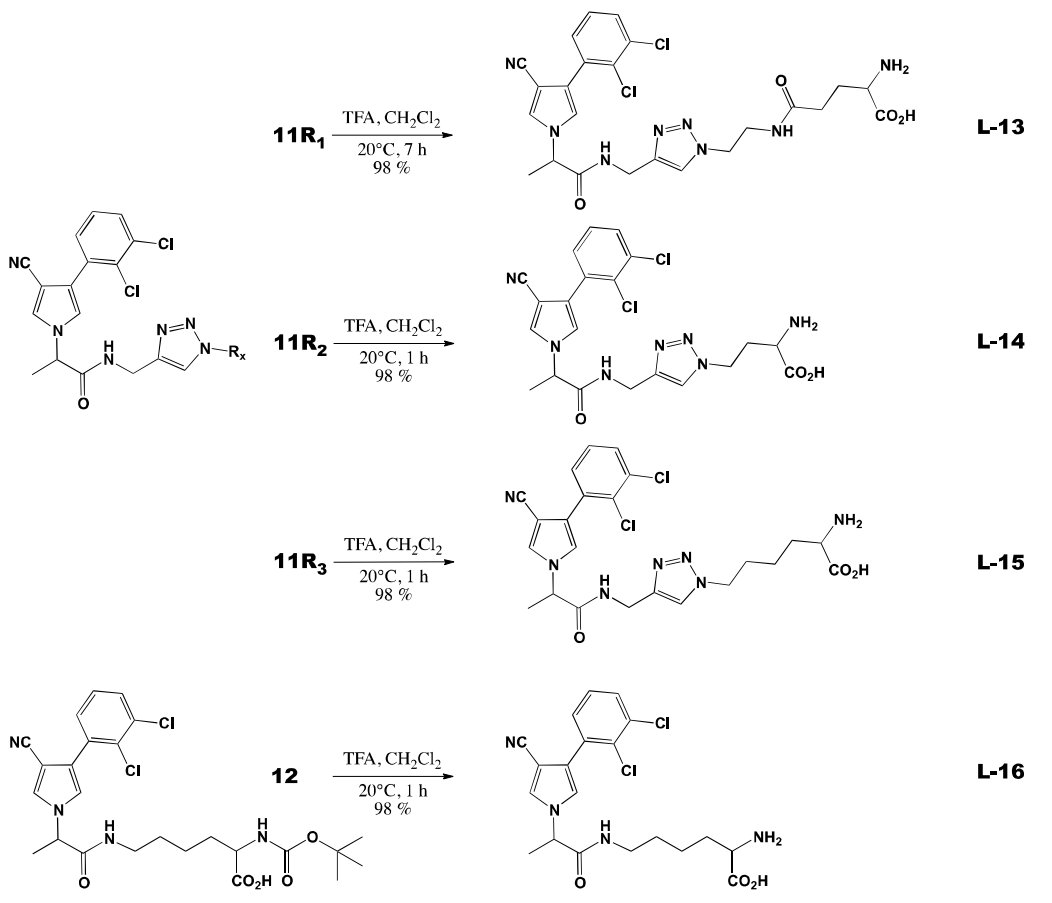

Figure 2. General reaction scheme showing the different steps of the synthesis of the amino acid-fungicide derivatives. DMAP: 4-Dimethylaminopyridine; EDCl: 1-Ethyl-3-(3dimethylaminopropyl)carbodiimide hydrochloride; $T F A$ : Trifluoroacetic acid 

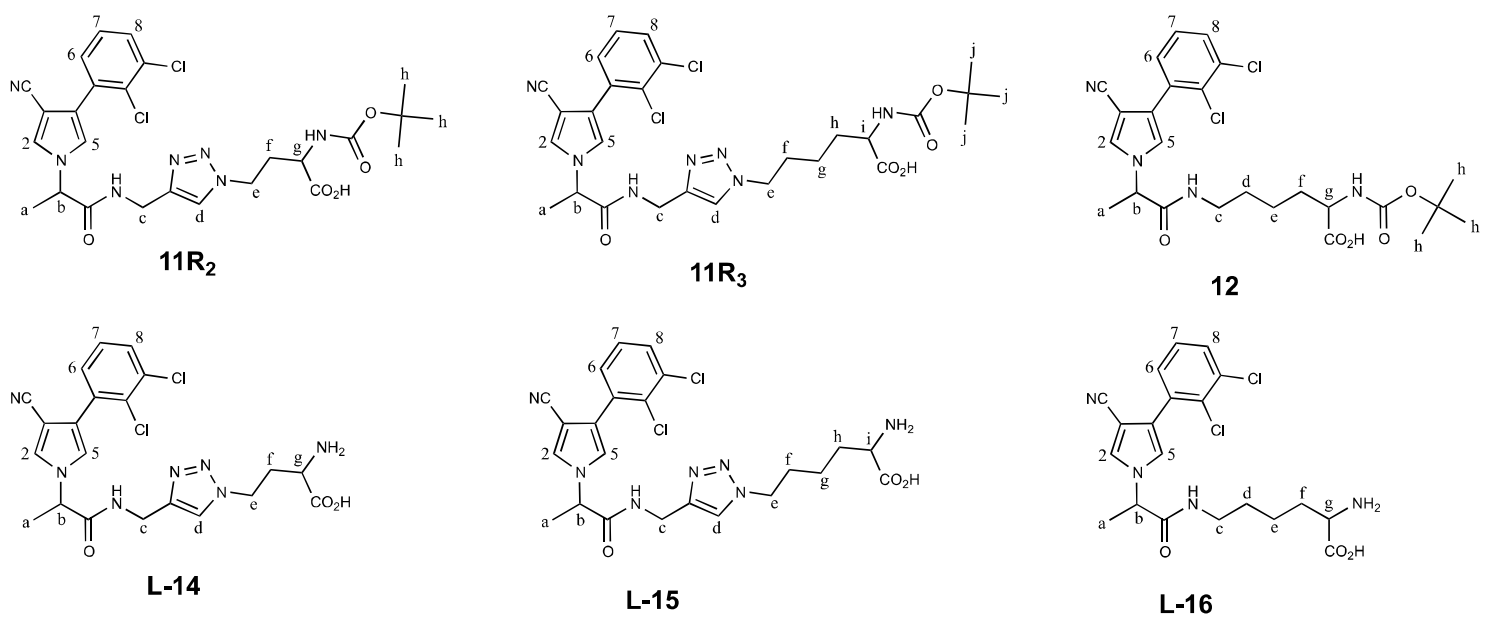

Figure 3. Fenpiclonil derivatives numbering for ${ }^{1} \mathrm{H}$ and ${ }^{13} \mathrm{C}$ assignments 


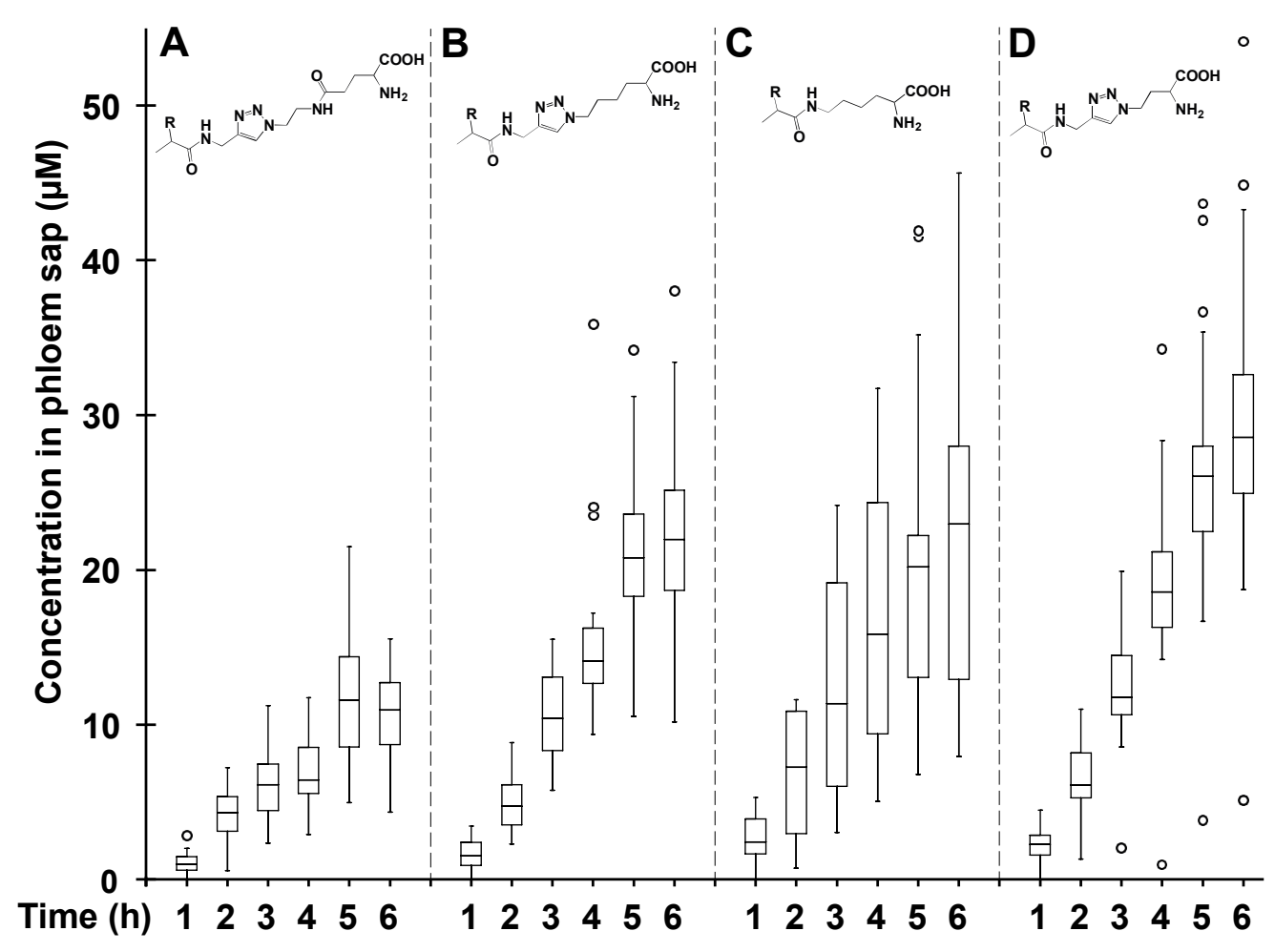

Figure 4. Time course of amino acid conjugates L-13 (A), L-15 (B), L-16 (C) and L-14 (D) concentration in phloem sap of Ricinus. Cotyledons were incubated in a standard buf fered solution at $\mathrm{pH} 5.0$ (Rocher et al, 2006) for $30 \mathrm{~min}$, then in the same solution containing compounds L-13, L-15, L-16 or L-14 at $100 \mu \mathrm{M}$ concentration. After $30 \mathrm{~min}$, the hypocotyl was severed in the hook region and then the sap was collected ever y hour for $6 \mathrm{~h}$. For box plots, $n=22(A), n=$ $18(\mathrm{~B}), \mathrm{n}=20$ (C and $\mathrm{D}) . \mathrm{R}=$ fenpiclonil. 


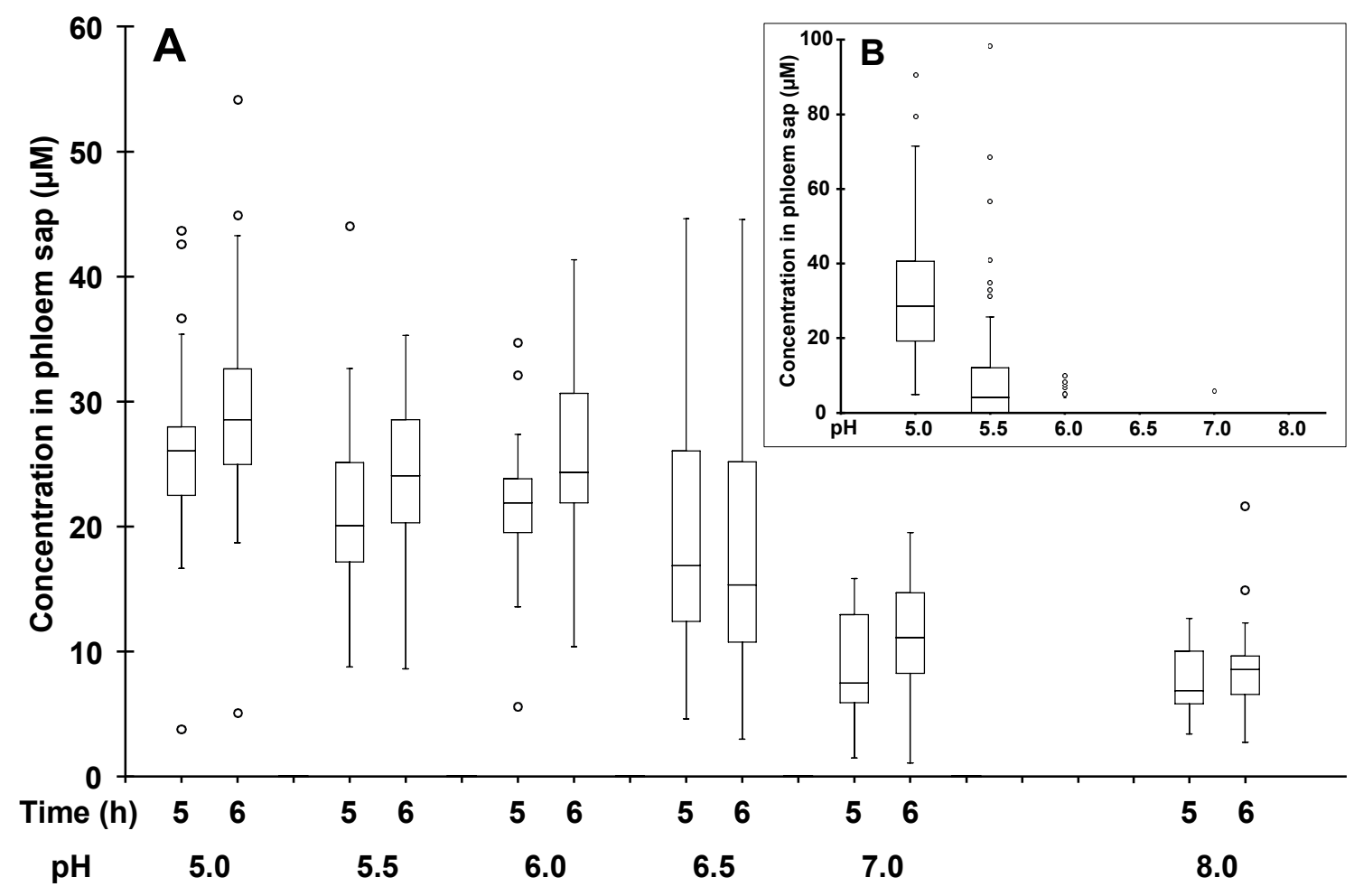

Figure 5. Concentration of amino acid conjugate L-14 (A) or acidic derivative 9 (B) in phloem sap of Ricinus as a function of the $\mathrm{pH}$ of the incuba tion medium. Cotyledons were incubated in a standard buffered solution at pH 5.0, 5.5, 6.0, 6.5, 7.0 or 8.0 (Rocher et al, 2006) for $30 \mathrm{~min}$, then in the same solution containing compound L-14 or 9 at $100 \mu \mathrm{M}$ concentration. After 30 min, the hypocotyl was severed in the hook region and then the sap was collected when the concentration reach a plateau, ie after 5 and 6 hours $(\mathrm{A})$ or during the third and fourth hours of incubation (B). For box plots, $\mathrm{n}=20$ for all $\mathrm{pHs}(\mathrm{A}), \mathrm{n}=58(\mathrm{pH} 5.0), \mathrm{n}=88(\mathrm{pH} 5.5), \mathrm{n}=39(\mathrm{pH} 6.0), \mathrm{n}=22$ $(\mathrm{pH} 6.5), \mathrm{n}=12(\mathrm{pH} 7.0)$ and $\mathrm{n}=11(\mathrm{pH} 8.2)(\mathrm{B})$. 


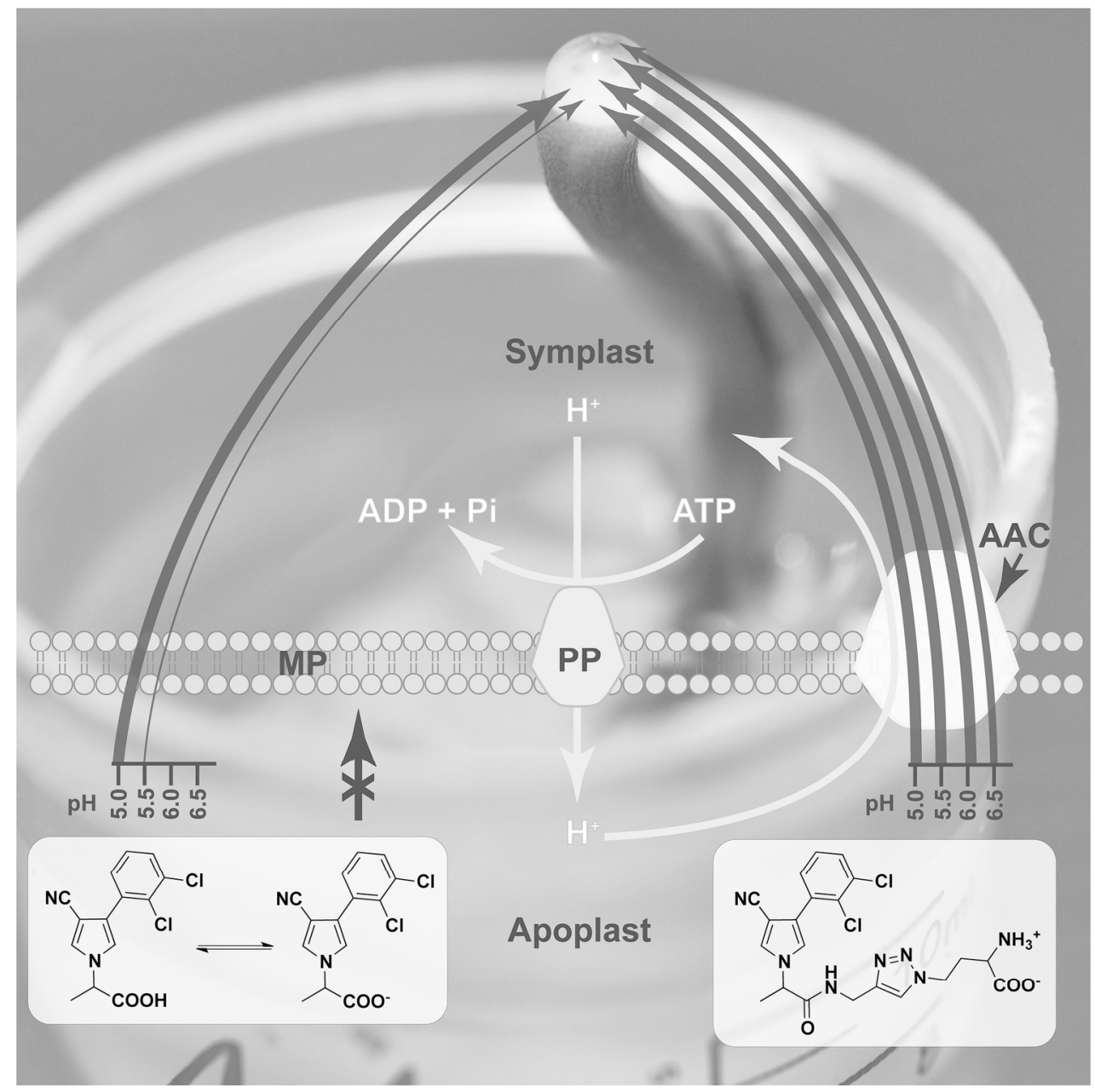

Figure 6. Phloem systemicity dependence of the acidic derivative (9) and the L- $\alpha$-amino acid conjugate (L-14) of fenpiclonil at apoplastic $\mathrm{pH}$ values (from $\mathrm{pH} 5.0$ to 6.5). The thickness of each arrow is related to the concentrations of both compounds found in the phloem sap. AAC: amino acid carrier; MP: plasma membrane; PP: proton pump. 
Table 1. Chromatographic data for tested products

\begin{tabular}{|c|c|c|c|c|c|c|}
\hline \multicolumn{3}{|c|}{ Mobile Phase (gradient) } & \multirow{2}{*}{$\begin{array}{c}\text { Delivery } \\
\left(\mathrm{ml} \cdot \mathrm{min}^{-1}\right)\end{array}$} & \multirow{2}{*}{$\begin{array}{c}\text { Detection UV } \\
(\mathbf{n m})\end{array}$} & \multirow[t]{2}{*}{ Compound } & \multirow{2}{*}{$\begin{array}{l}\text { Retention Time } \\
\text { (min) }\end{array}$} \\
\hline Time (min) & Water + TFA $0.1 \%$ & $\mathrm{CH}_{3} \mathrm{CN}$ & & & & \\
\hline \multirow{2}{*}{$\mathrm{t}=0$} & \multirow{2}{*}{70} & \multirow{2}{*}{30} & \multirow{6}{*}{0.8} & \multirow{6}{*}{218} & Fenpiclonil & 24.6 \\
\hline & & & & & 9 & 22.9 \\
\hline $\mathrm{t}=30$ & 40 & 60 & & & L-13 & 8.0 \\
\hline $\mathrm{t}=35$ & 70 & 30 & & & L-14 & 8.8 \\
\hline \multirow{2}{*}{$\mathrm{t}=37$} & \multirow{2}{*}{70} & \multirow{2}{*}{30} & & & L-15 & 9.7 \\
\hline & & & & & L-16 & 9.2 \\
\hline
\end{tabular}




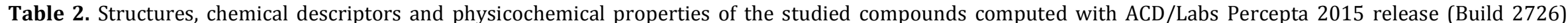

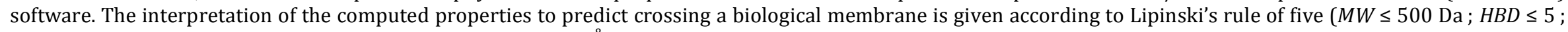

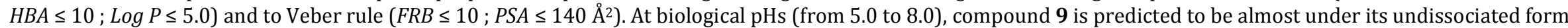
$(\mathrm{pKa}=3.58 \pm 0.40)$ and compounds L-13 $-\mathbf{L - 1 6}$ are predicted to be under their zwiterrionic form.

$M W$ : molecular weight ; $H B D$ : number of hydrogen bond donors ; $H B A$ : number of hydrogen bond acceptors ; FRB : free rotatable bonds ; $P S A$ : polar surface area.

\begin{tabular}{|c|c|c|c|c|c|c|c|c|c|c|}
\hline No & Structure & MW & HBD & HBA & $\begin{array}{c}\log D \\
(p H 5.0)\end{array}$ & $\begin{array}{c}\log D \\
(\mathrm{pH} \mathrm{8.0})\end{array}$ & FRB & PSA $\left(\AA^{2}\right)$ & $\begin{array}{c}\text { Lipinski's } \\
\text { rule of five } \\
\text { violation } \\
(*) \\
\end{array}$ & $\begin{array}{c}\text { Veber rules } \\
\text { violation } \\
(* *)\end{array}$ \\
\hline $\begin{array}{l}\text { Fenpi } \\
\text { clonil }\end{array}$ & & 237.08 & 1 & 2 & 3.92 & 3.92 & 2 & 39.58 & $0 / 4$ & $0 / 2$ \\
\hline 9 & $\mathrm{Cl}$ & 309.15 & 1 & 4 & 2.05 & -0.45 & 4 & 66.02 & $0 / 4$ & $0 / 2$ \\
\hline
\end{tabular}

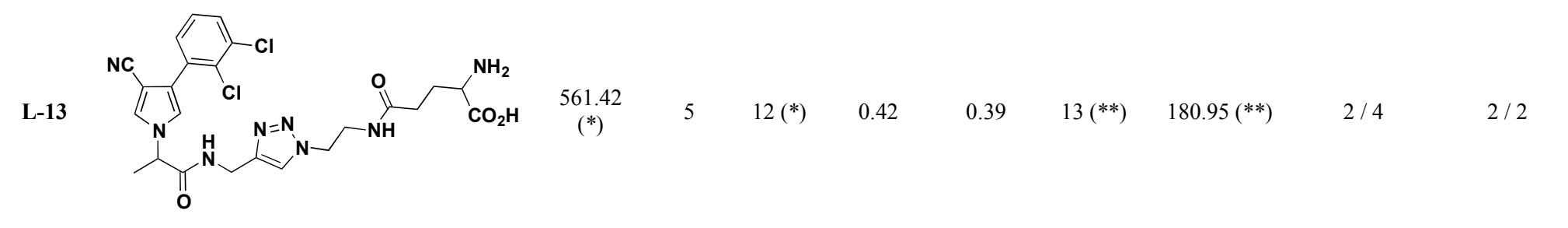




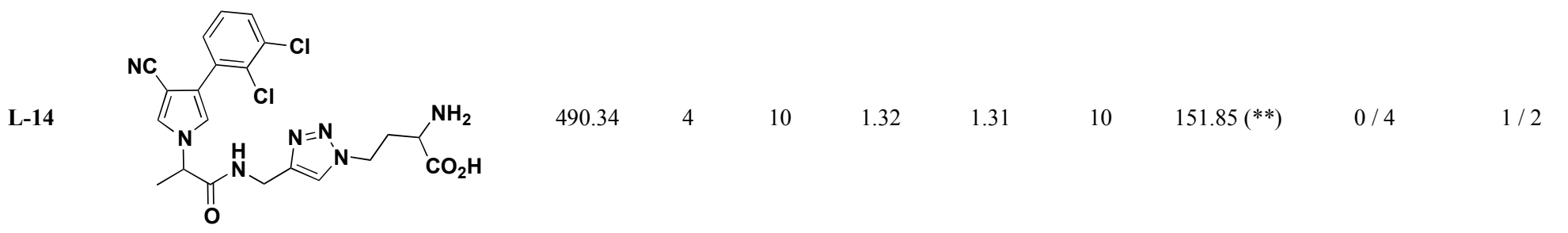

(*)

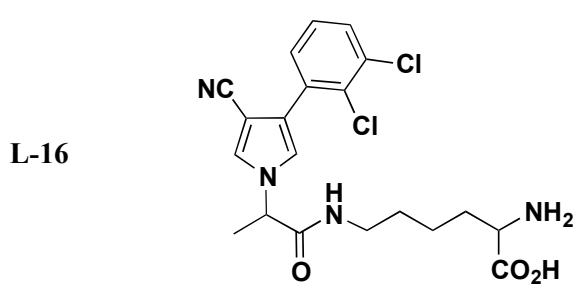

$\begin{array}{lllllllll}437.32 & 4 & 7 & 1.98 & 1.97 & 10 & 121.14 & 0 / 4 & 0 / 2\end{array}$

